\title{
Coupled rotational dynamics of Jupiter's thermosphere and magnetosphere
}

\author{
C. G. A. Smith ${ }^{1, *}$ and A. D. Aylward ${ }^{1}$ \\ ${ }^{1}$ Atmospheric Physics Laboratory, Department of Physics and Astronomy, University College, London, UK \\ * now at: Teesdale School, Barnard Castle, County Durham, UK
}

Received: 16 March 2008 - Revised: 13 September 2008 - Accepted: 2 December 2008 - Published: 13 January 2009

\begin{abstract}
We describe an axisymmetric model of the coupled rotational dynamics of the thermosphere and magnetosphere of Jupiter that incorporates self-consistent physical descriptions of angular momentum transfer in both systems. The thermospheric component of the model is a numerical general circulation model. The middle magnetosphere is described by a simple physical model of angular momentum transfer that incorporates self-consistently the effects of variations in the ionospheric conductivity. The outer magnetosphere is described by a model that assumes the existence of a Dungey cycle type interaction with the solar wind, producing at the planet a largely stagnant plasma flow poleward of the main auroral oval. We neglect any decoupling between the plasma flows in the magnetosphere and ionosphere due to the formation of parallel electric fields in the magnetosphere. The model shows that the principle mechanism by which angular momentum is supplied to the polar thermosphere is meridional advection and that mean-field Joule heating and ion drag at high latitudes are not responsible for the high thermospheric temperatures at low latitudes on Jupiter. The rotational dynamics of the magnetosphere at radial distances beyond $\sim 30 R_{J}$ in the equatorial plane are qualitatively unaffected by including the detailed dynamics of the thermosphere, but within this radial distance the rotation of the magnetosphere is very sensitive to the rotation velocity of the thermosphere and the value of the Pedersen conductivity. In particular, the thermosphere connected to the inner magnetosphere is found to super-corotate, such that true Pedersen conductivities smaller than previously predicted are required to enforce the observed rotation of the magnetosphere within $\sim 30 R_{J}$. We find that increasing the Joule heating at high latitudes by adding a component due to rapidly fluctuating electric fields is unable to explain the
\end{abstract}

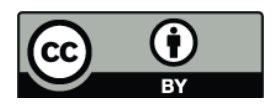

Correspondence to: C. G. A. Smith (cgasmith@gmail.com) high equatorial temperatures. Adding a component of Joule heating due to fluctuations at low latitudes is able to explain the high equatorial temperatures, but the thermospheric wind systems generated by this heating cause super-corotation of the inner magnetosphere in contradiction to the observations. We conclude that the coupled model is a particularly useful tool for study of the thermosphere as it allows us to constrain the plausibility of predicted thermospheric structures using existing observations of the magnetosphere.

Keywords. Magnetospheric physics (Magnetosphereionosphere interactions; Planetary magnetospheres) Meteorology and atmospheric dynamics (Thermospheric dynamics)

\section{Introduction}

The magnetospheres of Jupiter and Saturn are dominated by the influence of the planets' rapid rotation frequencies. The plasma in the magnetospheres of both planets exhibit partial corotation (McNutt et al., 1979; Richardson, 1986), indicating that angular momentum has been transferred from the planet. This angular momentum transfer occurs because of the presence of a conducting layer in the atmosphere, with which the magnetosphere may interact. This conducting region occurs within the ionosphere, which is colocated with the (neutral) thermosphere, and it is the rotation of this region of the neutral atmosphere, not the deep rotation velocity, that directly controls the magnetosphere. In the case of Jupiter, on which we focus here, this corresponds to pressures lower than $\sim 2$ microbar (altitudes greater than $\sim 300 \mathrm{~km}$ above the 1 bar level).

The importance of the thermospheric rotation velocity, as distinct from the deep rotation velocity, has long been recognised in magnetospheric studies. There are very few measurements of thermospheric winds at either Jupiter or Saturn

Published by Copernicus Publications on behalf of the European Geosciences Union. 
(e.g. Gladstone et al., 2005), which are insufficient to empirically determine the appropriate rotation velocity. To cope with this lack of information, a simple theoretical model of magnetosphere-thermosphere coupling was developed for Jupiter by Huang and Hill (1989) and later improved by Pontius (1995). This model assumed that the principal process by which angular momentum is transported within the thermosphere was vertical viscous transport. This yielded a linear relationship between the thermospheric and magnetospheric rotation velocities that allowed the unknown dynamics of the thermosphere to be simply parameterised using an "effective" ionospheric conductivity. Although developed for Jupiter, the effective conductivity model has also been adopted for the Saturn case (e.g. Cowley and Bunce, 2003; Saur et al., 2004).

Recently, this model has been critically analysed for the case of Saturn by Smith and Aylward (2008). This study reached four main conclusions:

1. Meridional advection, not vertical viscous transport, is the principal mechanism for transporting angular momentum to the high latitude thermosphere.

2. As a result, the effective conductivity model of Huang and Hill (1989) is a poor parameterisation of the thermospheric rotation velocity.

3. Meridional advection of angular momentum produces meridionally smoothed structures in the thermospheric rotation velocity which feed back on the rotational structure of the magnetosphere.

4. Super-corotation of the neutral atmosphere arises at latitudes coupled to the inner magnetosphere. This may lead to super-corotation of the inner magnetosphere itself.

These conclusions represent a new perspective on the thermosphere-magnetosphere interaction, in which meridional advection of angular momentum within the thermosphere is as important for the rotational structure as radial diffusion of angular momentum in the magnetosphere. As a result rotational structures are influenced by the complexity of both systems, and develop mutually, rather than the magnetosphere imprinting its rotational structure on an essentially passive thermosphere.

While the basic physics of angular momentum transfer is essentially identical at both Jupiter and Saturn, there are several important differences that influence the character of magnetosphere-thermosphere coupling. Jupiter's magnetic field is an order of magnitude stronger than Saturn's, resulting in a much larger magnetosphere. At Jupiter internal mass-loading is dominated by a single source, Io, which injects material at a rate of approximately $1000 \mathrm{~kg} / \mathrm{s} \mathrm{(e.g.}$ Delamere and Bagenal, 2003). At Saturn internal massloading is distributed between a number of moons and rings, resulting in a complicated plasma distribution and a lower rate of mass-loading of the order of $\sim 40 \mathrm{~kg} / \mathrm{s}$ (Richardson et al., 1998). The greater size and rate of mass-loading of the Jovian magnetosphere means that magnetospherethermosphere coupling currents must be stronger in the sense that they transfer a greater quantity of angular momentum.

The region of Jupiter's thermosphere from which this greater quantity of angular momentum is extracted is expected to have a higher column mass than the equivalent region at Saturn. This is because the peak of the Pedersen conductivity corresponds approximately to the altitude where the ion-neutral collision frequency and ion gyrofrequency are equal. The higher magnetic field at Jupiter (by a factor of $\sim 10$ ) means a higher gyrofrequency, so, since the collision frequency is proportional to neutral density, this equality occurs in a proportionately denser layer of the atmosphere. This effect is compensated for by the higher gravitational field strength at Jupiter's surface (by a factor of $\sim 2$ ) which reduces the scale height, so that the vertical width of the peak of the Pedersen conductivity is proportionately reduced. Overall, therefore, we can estimate that the column mass of thermosphere from which angular momentum is extracted is approximately 5 times greater at Jupiter than at Saturn.

This means that, all other things being equal, a smaller proportion of the angular momentum present in that layer of the thermosphere will be removed per second, and atmospheric advection and viscosity should be able to replace it more rapidly. However, as commented above, the rate of angular momentum transfer is much greater at Jupiter than at Saturn, in which case the thermosphere at Jupiter is expected to be able to replace extracted angular momentum relatively less efficiently. While these simple considerations illustrate some of the possible differences between the response of the thermosphere to magnetospheric forcing at Jupiter and Saturn, many other factors must be included to understand the full picture, including the precise distribution of conductivity, the mapping of field lines between the thermosphere and magnetosphere, and the relative magnitude of the plasma flows in the magnetosphere. It is to include all these factors simultaneously that we require a numerical model. Note that the purpose of this paper is to examine magnetospherethermosphere coupling at Jupiter only; we hope to present a comparison of the two planets in a future study.

A related problem at both planets is the high thermospheric temperature. At Jupiter, the observed equatorial neutral temperatures of $\sim 900 \mathrm{~K}$ (Seiff et al., 1998) are well in excess of those expected if absorption of solar EUV is the primary energy source. Spectroscopic measurements of the temperature of the $\mathrm{H}_{3}^{+}$molecular ion have shown that the high latitude upper atmosphere temperatures are also high, in the region of $\sim 700-1250 \mathrm{~K}$ (Lam et al., 1997; Stallard et al., 2002). The source of the energy required to produce high temperatures at low latitides remains a mystery. It has been proposed that these globally high temperatures may be explained by the injection of energy from the magnetosphere at 
high latitudes that is subsequently redistributed globally by equatorward winds (Waite et al., 1983; Atreya, 1986; Miller et al., 2000; Bougher et al., 2005; Smith et al., 2005a; Melin et al., 2006).

Similar high temperatures are present in Saturn's thermosphere, and recent studies have attempted to explain these measurements by the redistribution of high latitude heating. Smith et al. (2005b) found that an arbitrary high latitude source of thermal energy did generate sufficient redistributive winds to reproduce the observed temperatures, but the more sophisticated calculations of Smith et al. (2007) showed that when high-latitude energy inputs of Joule heating and ion drag were included, poleward meridional winds were generated that cooled, rather than heated, low latitudes.

The results of Smith et al. (2007) and Smith and Aylward (2008), pertaining to thermospheric temperature and angular momentum transfer, respectively, were both generated using the same simplified model of Saturn's thermosphere. In this paper we describe an application of the same modelling techniques to Jupiter, with the advantage that we are able to make use of an existing physical model of Jupiter's middle magnetosphere (Nichols and Cowley, 2004), allowing us to selfconsistently couple models of the thermosphere and magnetosphere.

We also investigate a possible additional thermospheric energy source that has been raised for Earth (Codrescu et al., 1995) and more recently for Jupiter and Saturn (Smith et al., 2005a) but not yet tested: the possibility that small scale fluctuations in the electric field may increase the total Joule heating and thus account for the energy deficit. Our model not only allows us to examine this question - it also allows us to test the consistency of such a situation with existing measurements of the magnetosphere.

In Sect. 2 we describe the background observations and theory necessary to understand our model; in Sects. 3-6 we describe respectively our magnetosphere, thermosphere and ionosphere models and how they are coupled together. Our initial results are described in Sect. 7 and the results of forcing the model with extra Joule heating from electric field fluctuations in Sect. 8. In Sects. 9 and 10 we discuss our results and conclude.

\section{Theoretical background}

\subsection{Magnetosphere-thermosphere coupling at Jupiter}

In Jupiter's magnetosphere the dominant internal source of plasma is volcanism on the moon Io, which orbits Jupiter every $\sim 40 \mathrm{~h}$ at approximately $6 R_{J}$. Clouds of sulphur and oxygen ejected from Io form a vast torus of neutral gas close to the equatorial plane. Some of this neutral gas subsequently becomes ionised and is then under the influence of the planet's magnetic field.

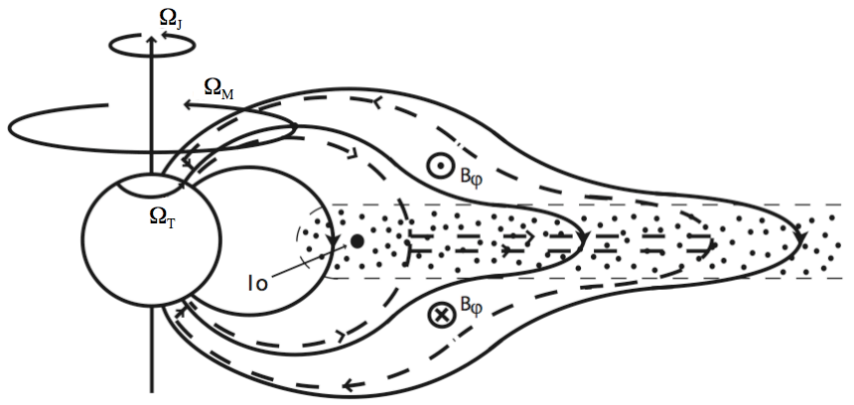

Fig. 1. Schematic diagram of Jupiter's inner and middle magnetosphere (adapted from Cowley and Bunce, 2001). Solid lines are magnetic field lines; dashed lines are the corotation enforcement currents.

The newly created plasma in the Io torus feels a torque exerted on it by the planet's upper atmosphere which accelerates it towards corotation with the upper atmosphere. The upper atmosphere feels an equal and opposite anti-corotational torque. In steady state it is supposed that the upper atmosphere is viscously and convectively coupled to the deep atmosphere of Jupiter, and that this coupling supplies sufficient angular momentum to balance the anti-corotational torque from the plasma disk.

In the Io torus, this ionospheric torque is adequate to enforce almost perfect corotation of the plasma. However, the plasma is known to diffuse radially outwards, driven by centrifugal "interchange instabilities" (e.g Siscoe and Summers, 1981). This outward diffusion leads to the formation of a plasma disk in the equatorial plane of the magnetosphere (Fig. 1). At larger radial distances the angular momentum required to enforce corotation of the plasma disk is much greater than that required in the Io torus, and ultimately the ionosphere is unable to supply sufficient torque to enforce corotation.

The result is that the plasma disk in the middle magnetosphere and the magnetically connected upper atmosphere are both expected to sub-corotate with respect to the deep planetary angular velocity $\Omega_{J}$, at angular velocities of $\Omega_{M}$ and $\Omega_{T}$ respectively, where, initially, we expect $\Omega_{M} \leq \Omega_{T} \leq \Omega_{J}$. The plasma in the disk continues to diffuse radially outwards and is eventually lost from the magnetosphere, by processes which are not well understood. Thus the plasma disk slowly extracts angular momentum from the planetary rotation.

Hill (1979) constructed a simple physical model of this situation, assuming a constant rate of plasma outflow, a dipolar field and a uniform ionospheric conductivity. This model was later developed to include thermospheric sub-corotation (Huang and Hill, 1989; Pontius, 1995), to take account of a non-dipolar magnetospheric field (Pontius, 1997), and to calculate the possible association between the plasma disk and Jupiter's main oval auroras (Hill, 2001; Cowley and Bunce, 2001). More recently, Nichols and Cowley (2004) 
have examined the effect of enhancements to the ionospheric conductivity associated with the main oval auroras. It is this model of the middle magnetosphere that we employ here. All of the studies mentioned above neglected the development of parallel electric fields in the magnetosphere that may decouple the plasma angular velocities in the upper atmosphere and magnetosphere. This possibility is discussed in Sect. 3.3.

\subsection{Magnetosphere-thermosphere coupling currents}

Throughout this study we simplify our modelling by assuming axisymmetry of the entire magnetosphere-atmosphere system. If we further assume during this initial discussion that the conducting region of the thermosphere-ionosphere is a thin sheet with a uniform rotational velocity $\Omega_{T}$ at each colatitude and the connected region of the magnetosphere has a uniform rotational velocity $\Omega_{M}$, then the appropriate electric field in the ionosphere is:

$E_{\theta}^{*}=\rho_{i}\left(\Omega_{T}-\Omega_{M}\right) B_{i}$

where $\rho_{i}$ is the perpendicular off-axis distance in the ionosphere, and we have assumed that the ionospheric magnetic field $B_{i}$ is vertical. This implies an equatorward-directed Pedersen current $J_{\theta}$ :

$J_{\theta}=\Sigma_{P} E_{\theta}^{*}=\Sigma_{P} \rho_{i}\left(\Omega_{T}-\Omega_{M}\right) B_{i}$

where $\Sigma_{P}$ is the height-integrated Pedersen conductivity of the ionosphere.

As shown in Fig. 1, to ensure continuity this current, which flows in both the Northern and Southern Hemispheres, must close in the equatorial plane of the magnetosphere, such that there is a radial current $J_{\rho}$ flowing away from the planet. The ionospheric current $J_{\theta}$ exerts an anticorotational (clockwise viewed from above the north pole) $\boldsymbol{J} \times \boldsymbol{B}$ torque on the ionosphere, to be discussed further below. An equal and opposite corotational torque acts on the plasma in the equatorial magnetosphere.

\subsection{Hall current}

In addition to the meridional Pedersen current we must also consider the meridional Hall current, which may be driven directly by meridional winds in the thermosphere, as discussed by Smith and Aylward (2008). This can be incorporated if we appropriately define $\Omega_{T}$ :

$\rho_{i} \Omega_{T}=\rho_{i} \Omega_{J}+U_{\phi}+\frac{\Sigma_{H}}{\Sigma_{P}} U_{\theta}$

where $U_{\phi}$ and $U_{\theta}$ are the eastward and southward thermospheric wind speeds respectively. Here $\Omega_{J}$ is the deep rotation velocity of the planet which is also the rotation velocity of the reference frame with respect to which the thermospheric wind speeds are defined.

If this definition of $\Omega_{T}$ is used in Eq. (2) then the contributions from Pedersen and Hall currents are accounted for.
Thus $\Omega_{T}$ is not strictly the rotation velocity of the neutrals it is an effective rotation velocity to which meridional winds may contribute. If the Hall conductivity is bigger than the Pedersen conductivity then the contribution from meridional winds may dominate. A more detailed discussion is given by Smith and Aylward (2008).

\subsection{Vertically extended ionosphere}

The above definition of $\Omega_{T}$ assumed that the ionosphere was a thin sheet. In practice, the ionosphere has vertical structure, such that each layer has a local effective rotation velocity $\omega_{T}$ which is completely analogous to that defined in Eq. (3)

$\rho_{i} \omega_{T}=\rho_{i} \Omega_{J}+u_{\phi}+\frac{\sigma_{H}}{\sigma_{P}} u_{\theta}$

where $\sigma_{P}, \sigma_{H}, u_{\phi}$ and $u_{\theta}$ are local values of the conductivites and neutral wind speeds respectively. To find the total current we just add the various layers in parallel, resulting in the following definition for $\Omega_{T}$ :

$\Sigma_{P} \Omega_{T}=\int \sigma_{P} \omega_{T} d z$

where $z$ is altitude and $\sigma_{P}$ is the local Pedersen conductivity at each layer so that

$\Sigma_{P}=\int \sigma_{P} d z$

Thus $\Omega_{T}$ represents a weighted average of the effective rotation velocity throughout the ionosphere; it is possible that no level of the thermosphere that is coupled to the magnetosphere actually physically rotates at this velocity.

\subsection{Effective conductivity}

Huang and Hill (1989) showed that if the angular momentum extracted from the thermosphere was replaced primarily by vertical viscous transfer, then for a given atmospheric structure the corotation lag of the thermosphere was a fixed proportion $K$ (our notation) of the corotation lag of the connected magnetosphere:

$\Omega_{J}-\Omega_{T}=K\left(\Omega_{J}-\Omega_{M}\right)$

which can be rearranged to give

$\Omega_{T}-\Omega_{M}=(1-K)\left(\Omega_{J}-\Omega_{M}\right)$

Substituting this into (Eq. 2), we have

$$
\begin{aligned}
J_{\theta} & =\Sigma_{P} \rho_{i}\left(\Omega_{T}-\Omega_{M}\right) B_{i} \\
& =\Sigma_{P} \rho_{i}(1-K)\left(\Omega_{J}-\Omega_{M}\right) B_{i} \\
& =\Sigma_{P}^{*} \rho_{i}\left(\Omega_{J}-\Omega_{M}\right) B_{i}
\end{aligned}
$$

where we have defined the effective conductivity

$\Sigma_{P}^{*}=\Sigma_{P}(1-K)$ 
The RHS of Eq. (9) does not contain $\Omega_{T}$, such that any explicit reference to the thermospheric rotation can be eliminated from calculations that refer to the magnetosphere alone. Note that to clearly distinguish the conductivity $\Sigma_{P}$ from the effective conductivity $\Sigma_{P}^{*}$ we will refer to it throughout this paper as the "true" conductivity.

It is tempting to interpret the effective conductivity as a harmless mathematical trick that combines the two unknown parameters $\Sigma_{P}$ and $\Omega_{T}$ into a single unknown $\Sigma_{P}^{*}$. However, it is important to emphasise that its usefulness is largely dependent on the special properties of the Huang and Hill model, in particular that the corotation lags of the thermosphere and magnetosphere are proportional (Eq. 7). If this model is not valid, then the quantity defined by Eq. (10) has limited physical meaning; we shall see in Sects. 7 and 8 that it behaves strangely if the inequality $\Omega_{M} \leq \Omega_{T} \leq \Omega_{J}$ is violated.

\section{Magnetosphere model}

The plasma flows in the Jovian magnetosphere are complex and not fully understood (see reviews by Khurana et al., 2004; Krupp et al., 2004). In particular, there is considerable debate concerning the structure of the outer regions of the magnetosphere, which couple to the solar wind and are connected to high magnetic latitudes at the planet (Kivelson and Southwood, 2005; McComas and Bagenal, 2007; Cowley et al., 2008; McComas and Bagenal, 2008).

Our model of the plasma flows in Jupiter's magnetosphere is a combination of the simple model of the whole magnetosphere presented by Cowley et al. (2005) and the more sophisticated model of the middle magnetosphere of Nichols and Cowley (2004). In outline, the model assumes that the rotation of the middle magnetosphere is controlled by outward diffusion of iogenic plasma balanced by angular momentum transfer from the planet (Hill, 1979). The motion of plasma at very large radial distances is described based on a solar wind interaction model first outlined by Cowley et al. (2003). This proposes that there is a significant quantity of open flux involved in a Dungey type interaction with the solar wind, mapping to a region of stagnant plasma flow of radius $\sim 10^{\circ}$ colatitude at the centre of the polar cap. The flow in the outer magnetosphere - lying between the two regions just described - is modelled as closed flux involved in the Vasyliūnas cycle and the return flow of the Dungey cycle.

Other models have been proposed for the structure of the outer jovian magnetosphere that describe a much weaker Dungey type interaction with the solar wind (Kivelson and Southwood, 2005; McComas and Bagenal, 2007). Both these papers suggest that the polar cap region of open flux is much smaller than that described by the Cowley model, but do not make alternative concrete quantitative statements about the plasma flows in the polar cap. We do not have a strong preference for the overall interpretation of plasma flow represented by the Cowley model: however we choose to employ it for

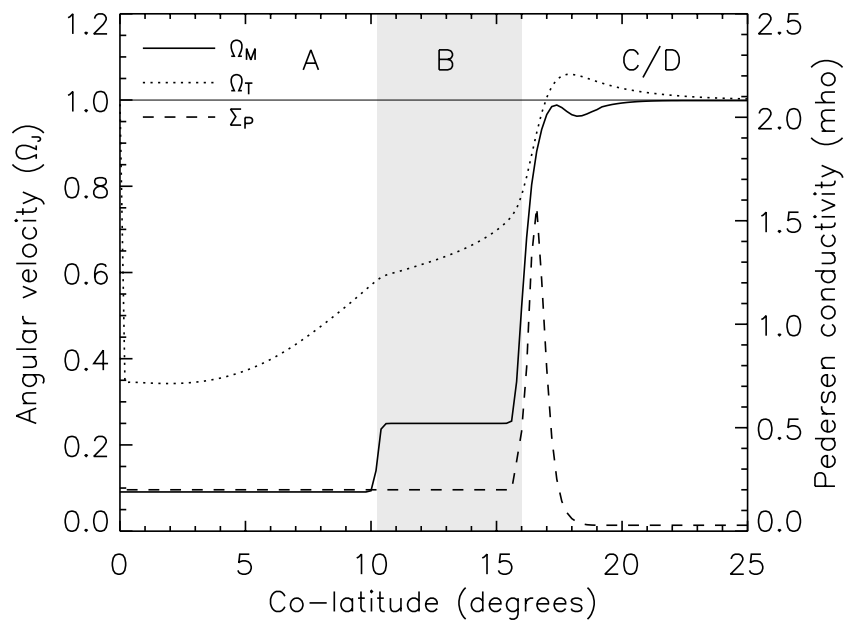

Fig. 2. Plasma velocity model for Jupiter, based on Nichols and Cowley (2004) and Cowley et al. (2005), mapped into the polar ionosphere (solid line). The shaded area denotes region $\mathrm{B}$. The profile of plasma velocity in regions A and B is fixed; the profile in regions $\mathrm{C}$ and $\mathrm{D}$ is calculated by the model. The profile shown is that calculated by our baseline model. Also shown are the heightintegrated Pedersen conductivities (dashed line) and neutral rotation velocities (dotted line) calculated by the baseline model (see Sect. 7).

the polar cap plasma flows because it provides a simple quantitative formulation that is easily integrated with our thermosphere model. In Sect. 9.1 we briefly discuss how our results might change if we used a model with a less stagnant (more rapidly rotating) plasma flow in the polar cap region.

The model assumes for simplicity that the magnetic field is axisymmetric, aligned with the planet's rotation axis, and north-south symmetric. The behaviour of the magnetosphere is then described in terms of the rotation velocities of axisymmetric shells of magnetic field lines. Each shell intersects the ionosphere at some colatitude $\theta$ and axial distance $\rho_{i}=R_{J} \sin \theta$ and the equatorial plane at some axial distance $\rho_{e}$, independent of longitude $(\phi)$.

The Cowley model may be split, conceptually, into four regions A-D. For regions A and B we use Cowley et al. (2005) and for regions C and D we use Nichols and Cowley (2004) (Fig. 2). These represent respectively the regions already discussed: regions that are open to the solar wind (A); regions of the outer magnetosphere involved in the Dungey and Vasyliūnas cycles (B); the sub-corotating middle magnetosphere $(\mathrm{C})$ and the corotating inner magnetosphere (D). These are the principal flow regions described by Cowley et al. (2005); we label them using the same letters as employed by Smith and Aylward (2008) in the context of Saturn. Note that the boundary between regions $\mathrm{C}$ and $\mathrm{D}$ is not welldefined, since the transition to perfect corotation is gradual. 


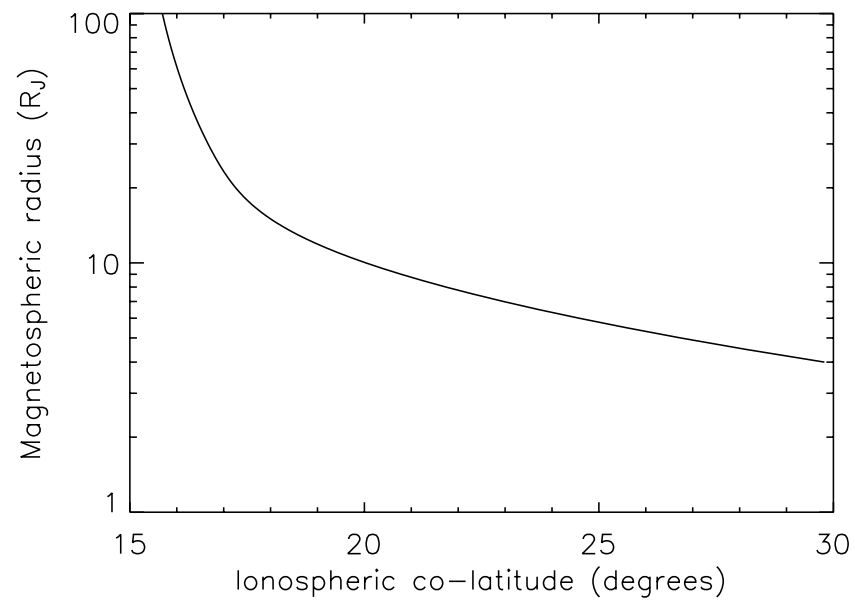

Fig. 3. Relation between ionospheric colatitude and magnetospheric radius implied by the Jovian magnetic field model.

\subsection{Open field and outer magnetosphere}

As already mentioned, we use the empirical model of Cowley et al. (2005) to represent the plasma flows in regions A and $\mathrm{B}$ of the magnetosphere. This model is specified according to co-latitude $\theta$ in the ionosphere:

$\Omega_{M}(\theta)=\omega_{A}+\frac{1}{2}\left[1+\tanh \left(\frac{\theta-\theta_{A B}}{\Delta \theta_{A B}}\right)\right]\left(\omega_{B}-\omega_{A}\right)$

Region A represents the region of "open" field lines associated with the tailward flow of the Dungey cycle. The colatitudinal extent of this region, which extends from the pole to $\theta_{A B}=10.25^{\circ}$, was determined by Cowley et al. (2005) through consideration of the amount of "open" flux expected in the system. A rotation velocity of $\omega_{A}=0.091 \Omega_{J}$ is chosen. This gives a rotation velocity close to zero, which is consistent with the theory of Isbell et al. (1984) if the effective conductivity is $0.2 \mathrm{mho}$ (we will discuss our adopted conductivities in more detail below). This "stagnant" behaviour is also consistent with the IR Doppler observations of Stallard et al. (2003). Region B is analagous to that described for Saturn, representing the Dungey cycle return flow and Vasyliūnas cycle. We use a value of $\omega_{B}=0.25 \mathrm{mho}$, which, in the formulation of Cowley et al. (2005), represents "expanded" conditions in which the magnetosphere is not overly compressed by the dynamic pressure of the solar wind.

Smith and Aylward (2008) found, in their study of Saturn, that using a fixed magnetosphere model similar to this produced anomalous behaviour and modified their plasma flow model such that, instead of the absolute plasma rotation velocity $\Omega_{M}$ being fixed in each region, the ratio of the plasma and neutral velocities $\chi=\Omega_{M} / \Omega_{T}$ was fixed instead. Since our main focus in this study is the structure of the physically self-consistent middle magnetosphere model, we will not implement such a modification here.

\subsection{Middle magnetosphere model}

\subsubsection{Summary of model}

Our middle magnetosphere model is closely based on the work of Nichols and Cowley (2004). In this section we firstly summarise the equations that constitute the Nichols and Cowley (2004) model and state our modifications. Further details of the model, in particular the magnetic field model and the origin of Eqs. (15) and (16) are given in Appendix A.

The relative simplicity of the model is underpinned by a series of simplifying assumptions. Firstly, we assume axisymmetry and north-south symmetry of both the planet and magnetosphere. For the planet, we believe this is justified as a first approximation. This will be discussed in Sect. 4.2. For the middle magnetosphere, this is a good approximation to the observed geometry (Nichols and Cowley, 2004).

Secondly, we assume sphericity of the conducting layer in the polar upper atmosphere and that it is permeated by a constant vertical polar B-field of magnitude $B_{i}=2 B_{J}$, where $B_{J}=426400 \mathrm{nT}$. This value was determined by Nichols and Cowley (2004) based on the VIP 4 internal field model of Connerney et al. (1998). Both of these assumptions are good first-order approximations to the observed geometry and magnetic field. Finally, we assume negligible fieldaligned potential drops in the magnetosphere. We will discuss this assumption in Sect. 3.3.

Since we are describing a physical model of the equatorial magnetosphere itself, it must be specified in terms of radial distance $\rho_{e}$ in the equatorial magnetosphere. We thus need a method of mapping rotation velocities along field lines between the equatorial magnetosphere and the high latitude ionosphere.

This is achieved using a "flux function" $F$. In this formulation, axially symmetric shells of magnetic field lines are defined by surfaces across which $F$ is constant. By separately defining this function in the ionosphere $\left(F_{i}\right)$ and magnetosphere $\left(F_{e}\right)$ it is then possible to map field lines along these shells by setting $F_{e}=F_{i}$. We employ identical flux functions to those of Nichols and Cowley (2004), as described in Appendix A; for further details the reader is referred to this paper and the references therein.

The resulting relationship between ionospheric colatitude and magnetospheric radius is plotted in Fig. 3. The most significant consequence of this mapping is that the region of the magnetosphere in the range $20-100 R_{J}$ maps to just $2^{\circ}$ of colatitude at the planet.

The plasma flow model itself is summarised by three equations. The first, which Nichols and Cowley (2004) refer to as the Hill-Pontius equation, is derived by balancing torques in the magnetosphere due to the outward diffusion of plasma with torques due to the magnetosphere-ionosphere coupling 
currents:

$$
\frac{1}{\rho_{e}} \frac{d}{d \rho_{e}}\left(\rho_{e}^{2} \Omega_{M}\right)=\frac{8 \pi \Sigma_{P}^{*} F_{e}\left|B_{z e}\right|}{\dot{M}}\left(\Omega_{J}-\Omega_{M}\right)
$$

Here $\rho_{e}$ is the radial distance from the centre of the planet in the equatorial plane of the middle magnetosphere; $\Omega_{M}$ is the angular rotation velocity of the plasma at that radius; $\Sigma_{P}^{*}$ is the effective Pedersen conductivity of the ionosphere (to be discussed further below); $F_{e}$ and $B_{z e}$ are the flux function and magnetic field in the magnetosphere respectively (fully defined in Appendix A); $\dot{M}$ is the mass outflow rate and $\Omega_{J}$ is the deep rotation velocity of the planet.

The second equation describes the magnitude of the upward field-aligned current in the ionosphere $j_{\| i}$ :

$$
j_{\| i}=\frac{4 B_{J}}{\rho_{e}\left|B_{z e}\right|} \frac{d}{d \rho_{e}}\left[\Sigma_{P}^{*} F_{e}\left(\Omega_{J}-\Omega_{M}\right)\right]
$$

In isolation, the field-aligned current specified by this second equation has no effect on the solutions of the first equation. However, in reality the field-aligned current should influence the angular rotation velocities since any particle precipitation related to large values of the upward field-aligned current is expected to enhance the effective ionospheric conductivity $\Sigma_{P}^{*}$. The third equation thus expresses this relationship:

$\Sigma_{P}^{*}=\Sigma_{P}^{*}\left(j_{\| i}\right)$

The functional form that is specified for this relationship influences the solutions of the first two equations, as discussed in some depth by Nichols and Cowley (2004). Details of our adopted functional form are given in Sect. 5.2.

Our principal modification to the model is to selfconsistently specify the neutral rotation velocity $\Omega_{T}$ using a model of the thermosphere. To include this, we must remove the effective conductivity $\Sigma_{P}^{*}$ and reintroduce $\Omega_{T}$. We thus substitute everywhere $\Sigma_{P}\left(\Omega_{T}-\Omega_{M}\right)$ for $\Sigma_{P}^{*}\left(\Omega_{J}-\Omega_{M}\right)$ to reintroduce $\Omega_{T}$ into Eqs. (12) and (13). We then replace Eq. (14) with a function that specifies the true conductivity $\Sigma_{P}$ in terms of the field-aligned current. Finally, we introduce a fourth equation which formally represents the thermosphere model by specifying $\Omega_{T}$ in terms of $\Omega_{M}$ and $\Sigma_{P}$ :

$$
\begin{aligned}
& \frac{1}{\rho_{e}} \frac{d}{d \rho_{e}}\left(\rho_{e}^{2} \Omega_{M}\right)=\frac{8 \pi \Sigma_{P} F_{e}\left|B_{z e}\right|}{\dot{M}}\left(\Omega_{T}-\Omega_{M}\right) \\
& j_{\| i}=\frac{4 B_{J}}{\rho_{e}\left|B_{z e}\right|} \frac{d}{d \rho_{e}}\left[\Sigma_{P} F_{e}\left(\Omega_{T}-\Omega_{M}\right)\right] \\
& \Sigma_{P}=\Sigma_{P}\left(j_{\| i}\right) \\
& \Omega_{T}=\Omega_{T}\left(\Omega_{M}, \Sigma_{P}\right)
\end{aligned}
$$

As we shall see below, $\Omega_{T}$ is not a simple function of $\Omega_{M}$ and $\Sigma_{P}$, but is calculated by a complex time-dependent numerical model. However, for a given distribution of $\Omega_{M}$ and $\Sigma_{P}$ there presumably exists at least one steady state of the thermosphere model. Ideally, we wish to run the thermosphere model towards steady state so that all four of the equations above represent steady state conditions. Our procedure for approaching steady state is described in Sect. 6.

\subsubsection{Method of obtaining solutions}

Our method of solution closely follows that employed by Nichols and Cowley (2004). The main difference is that we calculate a profile of $\Omega_{T}$ with radial distance based on the current state of our thermosphere model and employ this in Eqs. (15) and (16). We specify as our outer boundary condition at $100 R_{J}$ an azimuth-integrated radial current $I=100 \mathrm{MA}$, approximately equal to that determined from Galileo data (Khurana, 2001). We then select a value of the plasma rotation velocity $\Omega_{M}$ at the outer boundary and together with Eqs. (A4) and (17) this allows us to also calculate $j_{\| i}$ at the outer boundary. We then integrate Eqs. (15), (16) and (17) inwards from the outer boundary. Typically this initial choice diverges to very large negative or positive rotation velocities at small radial distances. We thus iterate our chosen value of $\Omega_{M}$ at the outer boundary until we obtain a solution that converges to the required inner boundary condition of near-rigid corotation at small radial distances.

The integration is specified using a double-precision FORTRAN subroutine, such that the magnetosphere model can easily be integrated with the existing FORTRAN thermosphere model. In practice, it is not possible to specify $\Omega_{M}$ with sufficient precision at the outer boundary to determine a solution that near-rigidly corotates at $\sim 4 R_{J}$. Instead, following Nichols and Cowley (2004), we employ an approximation in the inner region. This is based on the observation that at small radial distances the plasma is very close to rigid corotation such that $\Omega_{M} \approx \Omega_{T}$.

Firstly, we assume perfect corotation $\Omega_{M}=\Omega_{T}$ within $4 R_{J}$. At the planet, this corresponds to colatitudes greater than $30^{\circ}$. We then substitute the relation $\Omega_{M} \approx \Omega_{T}$ into Eq. (15) to yield the following approximation for $\Omega_{M}$ at radial distances slightly greater than $4 R_{J}$ :

$\Omega_{M} \simeq \Omega_{T}\left[1-\frac{\dot{M}}{4 \pi \Sigma_{P} F_{e}\left|B_{z e}\right|}\left(1+\frac{\rho_{e}}{2 \Omega_{T}} \frac{d \Omega_{T}}{d \rho_{e}}\right)\right]$

which is equivalent to Eq. (24) of Nichols and Cowley (2004) but with an extra term added to take account of the independent variability of $\Omega_{T}$ with radial distance. We use this formula to calculate an approximate value of $\Omega_{M}$ at a radial distance of $4 R_{J}$.

A similar expression can be determined for the fieldaligned current. We do not quote this (complicated) expression because its only practical use is the determination of $\Sigma_{P}$ in the inner region. When $\Sigma_{P}$ is calculated with this expression across the range $4-12 R_{J}$ is found to be always very close to the background value $\Sigma_{P 0}$ (to be defined in Sect. 5.2). Given this approximate profile of $\Sigma_{P}$ in the range $4-12 R_{J}$ and our approximate value of $\Omega_{M}$ at $4 R_{J}$, we integrate the exact formulation of Eq. (15) outwards with respect to radial distance to determine an approximate solution for $\Omega_{M}$ in the range $4-12 R_{J}$.

This approximate profile in the inner region then provides the convergence criteria for the integration from the outer 
boundary. When this integration reaches $12 R_{J}$, we compare the calculated value of $\Omega_{M}$ and its radial gradient to the equivalent values calculated from the inner region approximation at this radius. If the absolute values match within $1 \%$ of the inner region value and the gradients within $50 \%$ of the inner region value then we are satisfied that the integration has sufficiently converged. In practice, the latter condition is probably not necessary, since, if our inner region approximation is sufficiently accurate, a solution that matches within $1 \%$ of $\Omega_{M}$ would be expected to match the gradient within a narrower margin than $50 \%$. This is indeed the case: if the curves shown in Figs. 10 and 11 are examined at a radius of $12 R_{J}$ a small kink is apparent where the inner and outer region solutions join. It is clear that the gradients at this join, although not perfectly matched, differ by much less than $50 \%$.

We have validated this method by finding solutions corresponding to the assumptions of Nichols and Cowley (2004) and comparing these solutions to their results. We find that our method produces solutions closely matching those of the earlier study.

\subsection{Parallel electric fields}

The model of the middle magnetosphere that we have adopted from Nichols and Cowley (2004) makes the important simplifying assumption that plasma flows in the equatorial plane of the magnetosphere map exactly to magnetically connected plasma flows in the ionosphere. In reality this assumption partially breaks down because in order for the fieldaligned currents implied by the model to flow it is necessary for parallel electric fields to form along the magnetic field lines connecting the thermosphere and magnetosphere (e.g Mauk et al., 2002). The presence of these parallel electric fields means that the magnetic field lines are not equipotentials and the plasma flows in the equatorial magnetosphere and ionosphere become partially decoupled. These fields form because at high altitudes there are insufficient current carriers such that thermal currents cannot provide the necessary current density (Su et al., 2003).

The practical consequence of this decoupling is that the flux is not frozen into the plasma in the region of parallel electric fields. The flux tubes are able to "slip" relative to each other, so that the plasma angular velocity in the ionosphere is closer to corotation than that in the magnetosphere. This reduces the magnitude of the magnetospherethermosphere coupling currents and hence the rate at which angular momentum is transferred from the planet. Accurately modelling this effect is beyond the scope of this study: our results should therefore be considered to be a baseline approximation in which this aspect of the interaction is neglected.

\section{Thermosphere model}

\subsection{Summary of thermosphere model}

To calculate $\Omega_{T}$ self-consistently as a function of the values of $\Omega_{M}$ and $\Sigma_{P}$ calculated from the magnetosphere model, we employ a global, three-dimensional numerical model of the thermosphere. The model employed for this study is largely identical to that described by Smith and Aylward (2008) for their study of Saturn's thermosphere. In particular, the core thermospheric equations and our formulation of Joule heating and ion drag are completely unchanged. We will describe here only those features that it has been necessary to alter. We have incorporated elements from two existing Jupiter models - the JIM global three-dimensional model of the thermosphere and ionosphere Achilleos et al. (1998) and the Grodent et al. (2001) one-dimensional model of the auroral thermosphere and ionosphere.

We have, of course, changed the core parameters of the thermosphere model to those appropriate for Jupiter. The most of important of these is the planetary rotation frequency which we have set to $\Omega_{J}=1.76 \times 10^{-4} \mathrm{rad} \mathrm{s}^{-1}$, consistent with Nichols and Cowley (2004). We place the base of the model at a pressure $p_{0}=2 \mu$ bar $(300 \mathrm{~km}$ above the $1 \mathrm{bar}$ level) consistent with the JIM model (Achilleos et al., 1998). The temperature at the base of the model is set to a constant temperature of $262 \mathrm{~K}$, the temperature at $2 \mu \mathrm{bar}$ in the diffuse auroral model of Grodent et al. (2001). The winds at the base of the model are set to zero: thus the base of the model corotates with the planetary angular velocity $\Omega_{J}$.

The eddy coefficient in our baseline model - required in order to calculate eddy conduction and viscosity is set consistent with that of Grodent et al. (2001) at $K_{\tau}=1.4 \times 10^{2} \mathrm{~m}^{2} / \mathrm{s}$, placing the methane homopause at $\sim 1 \mu$ bar, just above the base of our model. Note that in our model $K_{\tau}$ does not vary with altitude. The mixing ratios of $\mathrm{H}, \mathrm{H}_{2}$ and $\mathrm{He}$ are taken from the diffuse auroral model of Grodent et al. (2001). These mixing ratios are fixed as a function of pressure. We do not calculate changes in these mixing ratios self-consistently because, since $\mathrm{H}_{2}$ is the dominant component throughout most of the thermosphere, the influence of composition changes upon the dynamics is relatively unimportant.

There is some flexibility in the boundary conditions that we specify. However, locating the lower boundary below the homopause, where vertical diffusive transport is dominated by eddy processes and hydrocarbon species are abundant, allows a double simplification. Firstly, strong eddy diffusion should keep the horizontal winds tightly coupled to those at lower altitudes. There are, to our knowledge, no measurements of significant horizontal winds in the mesosphere. However, the observed eastward speeds of zonal jets in the lower atmosphere are never greater than $\sim 150 \mathrm{~m} / \mathrm{s}$ (Ingersoll et al., 2004) globally and are less than $\sim 50 \mathrm{~m} / \mathrm{s}$ poleward of $45^{\circ}$ colatitude. Compared to the zonal plasma flow velocities 
of order $1000 \mathrm{~m} / \mathrm{s}$ close to the main auroral oval, these values are negligible and in any case we lack sufficient information to construct a reliable model of zonal winds at the lower boundary.

The second advantage of a lower boundary below the homopause is that infrared radiative cooling becomes important due to the high hydrocarbon densities. This process moderates the temperature, ensuring that the assumption of a relatively cool $(262 \mathrm{~K})$ fixed temperature lower boundary is reasonable. Again, there may be structure in the lower boundary temperature. For example, we would expect it to be somewhat warmer in regions subject to particle precipitation. However, we would need explicit models of radiative cooling and particle precipitation to accurately model such structures. The details of these processes are not the main focus of this study, so for simplicity it seems reasonable to assume a fixed temperature lower boundary.

In parallel with the magnetosphere model, the thermosphere model further assumes axial and north-south symmetry of the planet. These are much poorer assumptions for Jupiter than for Saturn because Jupiter's magnetic dipole is considerably tilted with respect to the rotation axis and offset with respect to the centre of the planet. This means that the structures of the magnetic north and south polar regions are very different and far from axisymmetric. However, axisymmetry is a useful first approximation that allows us to use a relatively high latitudinal resolution in our thermosphere model. This allows us to resolve the thermospheric behaviour close to the auroral oval in acceptable detail while maintaining manageable runtimes for our model. Possible consequences of our axisymmetry assumption are discussed in Sect. 4.2. Note that the assumption of axisymmetry essentially reduces the model from a three-dimensional model to a two-dimensional model in that the winds are assumed to be identical at all longitudes. However, the model still calculates three-component winds.

The horizontal resolution of our model is $0.2^{\circ}$ in latitude. Vertically, we use 30 pressure levels with a resolution of 0.4 pressure scale heights. We use a timestep of $3.0 \mathrm{~s}$.

\subsection{Symmetry assumptions}

As already mentioned, a major assumption of our thermosphere model is axisymmetry. The relatively small effect of solar forcing on the thermal structure and dynamics suggests that this component of axial asymmetry is not a major source of error in our calculations. However, the magnetic field of Jupiter is strongly asymmetric about the rotation axis, such that we must consider whether our symmetry assumptions seriously affect our calculations of magnetospherethermosphere coupling.

The observed location of the main auroral oval is a clear indication of the magnetic field asymmetry. The reference ovals provided by Grodent et al. (2003), based on UV imaging, show that the northern main oval does not normally en-

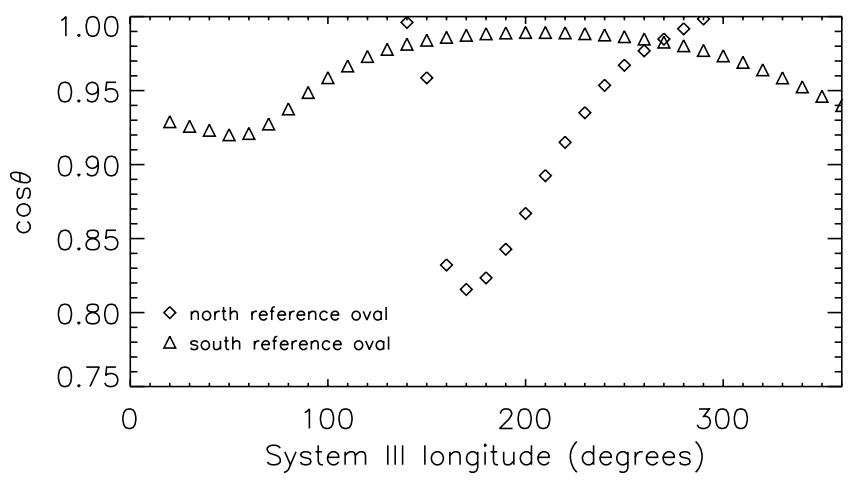

Fig. 4. Cosine of co-latitude plotted against longitude for the reference ovals of Grodent et al. (2003).

circle the rotation pole, lying wholly in the sector between system III longitudes of $\sim 130-300^{\circ}$. Within this range it forms an irregular oval shape that extends from just south of the rotational pole down to approximately $35^{\circ}$ colatitude. The southern oval is less irregular, forming an almost circular oval that encloses the southern rotational pole. It is, however, asymmetric about the pole, its colatitude varying in the range $8-14^{\circ}$.

To build an accurate model that incorporated these complexities, we would require a detailed magnetic field model that allowed us to map points in the middle magnetosphere directly onto these irregular ovals. An intermediate step that would capture most of the physics of the irregular ovals while maintaining simplicity of computation would be to use an offset tilted dipole model of the magnetic field. This is the type of magnetic field model employed by the JIM threedimensional model of the coupled thermosphere and ionosphere (Achilleos et al., 1998).

The resultant ovals in such a model are very nearly circular and offset from the rotational poles to a similar degree to the observed ovals. If we assume that the middle magnetosphere is axisymmetric then the plasma flow velocity along these ovals will be roughly constant in magnetic longitude. Thus, to first order, the principal difference between an axisymmetric and a non-axisymmetric model is that the circular region to which Joule heating and ion drag is applied is shifted away from the rotational pole. Thus the magnetospheric forcing of the thermosphere is essentially unchanged - only its location on the surface of the planet is altered.

The thermosphere responds to this forcing through a number of processes. Most of these, including effects due to thermal conduction, viscosity, advection, pressure gradients and the curvature of the planet (assuming perfect sphericity) are independent of latitude and longitude. The only process that is affected by the offset of the region of magnetospheric forcing is the Coriolis force. This is directly proportional to $\cos \theta$ where $\theta$ is the colatitude. Thus, in the northern oval, a thermospheric wind driven by ion drag in the region of the 
auroral oval close to the pole will experience a greater Coriolis force than a thermospheric wind driven by an identical ion drag at $35^{\circ}$ colatitude.

To first order, the $\cos \theta$ dependence of the Coriolis force is the only parameter that changes with the location of the region of magnetospheric forcing. Clearly, if the auroral oval was located at the equator, this would significantly alter the response of the thermosphere since the Coriolis force would be close to zero. However, within $35^{\circ}$ of the pole, the $\cos \theta$ factor reduces the Coriolis force by less than $20 \%$. Figure 4 shows this factor plotted against longitude for the reference ovals provided by Grodent et al. (2003). It is clear that in the north, the Coriolis force varies by no more than $\sim 20 \%$ within the colatitude range observed and in the south it varies by less than $\sim 10 \%$. These are therefore the order of magnitude errors that we expect the assumption of axisymmetry to introduce into our calculations of thermospheric winds. Given the numerous other simplifications and approximations involved in our modelling, these errors are not great and we expect that a non-axisymmetric model would produce very similar results to those presented here.

There are several caveats to the analysis above. Firstly, we have made the approximation of a perfectly spherical planet. This is a reasonable approximation, since even if the small oblateness of Jupiter were globally important, the polar regions themselves could be accurately approximated as part of a perfectly spherical surface.

Secondly, and more importantly, we have assumed that the only forcing is that due to magnetosphere-thermosphere coupling. There is of course a small forcing from absorption of solar radiation. The structures driven by solar radiation will interact with those driven by the magnetosphere. However, since solar driven effects are unable to explain the high thermospheric temperatures at Jupiter it seems certain that they have a relatively small influence on the thermal structure and therefore dynamics of the upper atmosphere, especially in the polar regions. It is also possible that strong zonal winds at the lower boundary or forcing by gravity waves may impose zonal structures from the lower and middle atmospheres on the thermosphere that interact with the magnetospheric forcing. In this case we might expect significantly different results in the non-axisymmetric case. However, there is no evidence that such forcing from below is of comparable magnitude to the magnetospheric forcing in the polar regions. There is some evidence for gravity waves in the thermosphere from the Galileo probe (Seiff et al., 1998), but this applies to the equatorial regions and the extent to which these gravity waves might transfer momentum to the upper atmosphere is not clear.

Thirdly, the predicted thermal and dynamical structure at the rotational equator clearly will be affected by an offset tilted dipole, since the tilt pushes part of both auroral ovals closer to the rotational equator. However, since the dipole is tilted by only $\sim 15^{\circ}$, the magnetic equator lies within $\pm 15^{\circ}$ of the rotational equator. Since the magnetic equator is ap- proximately equidistant from the auroral ovals in the offset tilted dipole model, we would expect it to correspond approximately to the equator in our axisymmetric model, with $\sim 20 \%$ modelling errors as discussed above. Thus, in our axisymmetric model, we expect the rotational equator to correspond to latitudes $<15^{\circ}$. Taking the band below $15^{\circ}$ latitude to represent the equator does not significantly change any of our conclusions regarding the equatorial thermal structure.

In summary, we think that our assumption of axisymmetry has only a small effect on our calculations of the thermospheric structure. This perhaps contradicts one's natural intuition that there is something "special" about the rotational pole. However, it should be remembered that in the rotating frame of the planet, the unforced thermosphere is a completely static shell of gas in hydrostatic equilibrium. In this idealised situation, the only observable difference between two locations is the magnitude and direction of the Coriolis force. Thus, when a circular region of magnetospheric forcing is applied anywhere at high latitudes, this, rather than the rotational pole, becomes the driving centre of the dynamics, and we expect the variations in the Coriolis force to introduce only small asymmetries into the thermospheric response.

Finally, note that north-south asymmetry arises largely as a consequence of the non-axisymmetric magnetic field. If we accept that axisymmetry is a reasonable assumption then the only remaining north-south asymmetry is the radius of the auroral oval. While neglecting this difference will have a small effect on our results, we do not expect it to have a large influence on the basic physics of angular momentum transfer that are the main topic of this study.

\section{Ionosphere model}

We do not include a self-consistent model of the Jovian ionosphere. Smith and Aylward (2008) justified this step for the Saturn case in terms of the poor reliability of gas giant ionosphere models. This justification also applies to Jupiter. Vertical profiles of electron density are available, from radio occultation data (Hinson et al., 1997, 1998) and more recently from telescopic observations (Lystrup et al., 2008). However, these profiles are spatially scattered and are not co-located with measurements of vertical thermospheric structure that would permit reliable calculations of ionospheric conductivity. Since global theoretical models of the ionosphere (e.g. Achilleos et al., 1998) have difficulty reproducing the available observations, it is apparent that the construction of a reliable global model of the Jovian ionosphere is a huge task in itself, and is thus a distraction from our main objective: to understand the coupled rotational dynamics of the thermosphere and magnetosphere.

Indeed, our intent is not to extend a fully-coupled Jovian thermosphere-ionosphere model such as those of Achilleos et al. (1998) and Bougher et al. (2005) to include a magnetosphere; rather our intent is to extend angular momentum 
transfer models such as those of Huang and Hill (1989) and Pontius (1995) to include a more sophisticated model of the thermosphere. It thus seems appropriate to maintain the latter authors' approach of a simple fixed model of the ionospheric conductivity.

We globally employ a conductivity model that is derived from a model of the auroral ionosphere. The principal reason for this is that the solution of the coupled equations that constitute the magnetosphere model is simplified considerably if the shape of the vertical profile of conductivity is constant globally. By "shape" we mean that, comparing two pressure levels, the relative conductivities are the same at all latitudes, even if the absolute conductivities vary with latitude. This simplification is discussed further in Sect. 5.3. We use an auroral conductivity model because most of the coupling with the magnetosphere occurs in regions with enhanced conductivity resulting from auroral processes. The vertical profile of this conductivity model and the horizontal profile of height-integrated conductivity are determined separately, as described below.

Finally, we also simplify our calculations of ionospheric conductivity by assuming a vertical magnetic field at all latitudes. This assumption is discussed further in Sect. 5.3.

\subsection{Vertical distribution}

For the vertical distribution we have chosen to use the 1-D auroral ionosphere model of Grodent et al. (2001) at all latitudes. The Grodent et al. model calculates auroral ion and electron densities and temperatures using a two-stream electron transport model. They present two versions of their model - a "diffuse" model intended to represent unstructured auroras in the polar cap and the afternoon sector of the main oval and a "discrete" model intended to represent the brighter, more structured aurora observed in the morning sector of the main oval. The models differ in the chosen input electron energy distributions which are determined so that the temperatures and emission signatures predicted by the model closely match a range of observational constraints derived from measurements of UV and IR emissions.

Both the diffuse and discrete models incorporate a double Maxwellian distribution with characteristic energies of $100 \mathrm{eV}$ and $3 \mathrm{keV}$. This component is largely responsible for heating and ionisation above the homopause. This is the region that contributes most significantly to the conductivity, since below the homopause the ionosphere is significantly depleted through charge exchange reactions with hydrocarbons. The models differ in the form of the high energy component, which has its greatest effect below the homopause, a region that has little influence on the conductivity. Thus, for our purposes, the two versions of the model yield very similar results. We choose to employ the diffuse model because we wish to represent the conductivity reasonably accurately across the whole of the main oval and polar cap: the diffuse

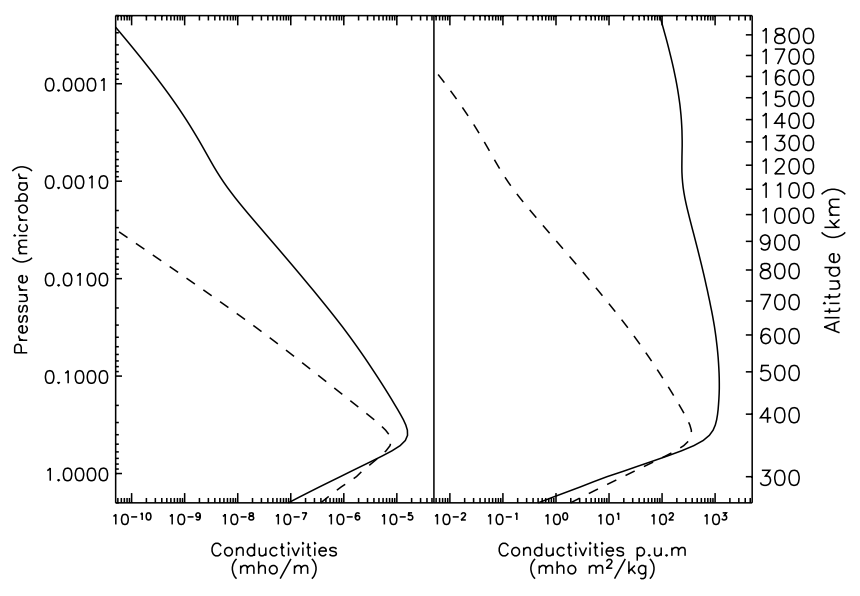

Fig. 5. Vertical distribution of conductivity. The left hand plot shows the local conductivities $\sigma_{P}$ (solid line) and $\sigma_{H}$ (dashed line) as a function of pressure calculated using the Grodent et al. (2001) $\mathrm{H}_{3}^{+}$densities and background atmosphere. The right hand plot shows the conductivities per unit mass $s_{P}$ and $s_{H}$, also calculated from the Grodent et al. model, using the same line formats. The altitude scale on the right hand side shows the altitude mapping in the Grodent et al. model; this does not apply to the results of our thermosphere model, which has a variable thermal structure.

model is clearly applicable over a greater proportion of this area.

The Grodent et al. model provides us with a single static profile of neutral densities and temperatures and $\mathrm{H}_{3}^{+}$and electron densities. To include this 1-D fixed ionosphere model in our 2-D time-variable thermosphere, our first step is simply to calculate values of $\sigma_{P}$ and $\sigma_{H}$ using the output of the Grodent et al. (2001) model. The expressions used to calculate these conductivities are given in Appendix B. The vertical conductivity distributions so calculated are shown in the left panel of Fig. 5. The pressure range shown corresponds to that covered by our thermosphere model. It is clear that the Pedersen conductivity is much more important than the Hall conductivity at almost all pressure levels. In particular, the peak Pedersen conductivity is several times greater than the peak Hall conductivity, such that the Pedersen dominates in a height-integrated sense. Thus the contribution of meridional winds to $\Omega_{T}$, as described by Eq. (3), is likely to be minimal.

The question then arises as to how one should apply these conductivity profiles, calculated using the specific thermal structure from the Grodent et al. model, to a thermosphere model that exhibits variable thermal structure. Our solution is to calculate, as a function of pressure, the quantities $s_{P}=\sigma_{P} / \rho$ and $s_{H}=\sigma_{H} / \rho$, where $\rho$ is the neutral mass density. We then use the same profiles of $s_{P}$ and $s_{H}$, as a function of pressure, at each latitude. The advantage of these quantities is that the height-integrated conductivities then depend only on $s_{P}$ and $s_{H}$, not on the thermal structure, since, for 


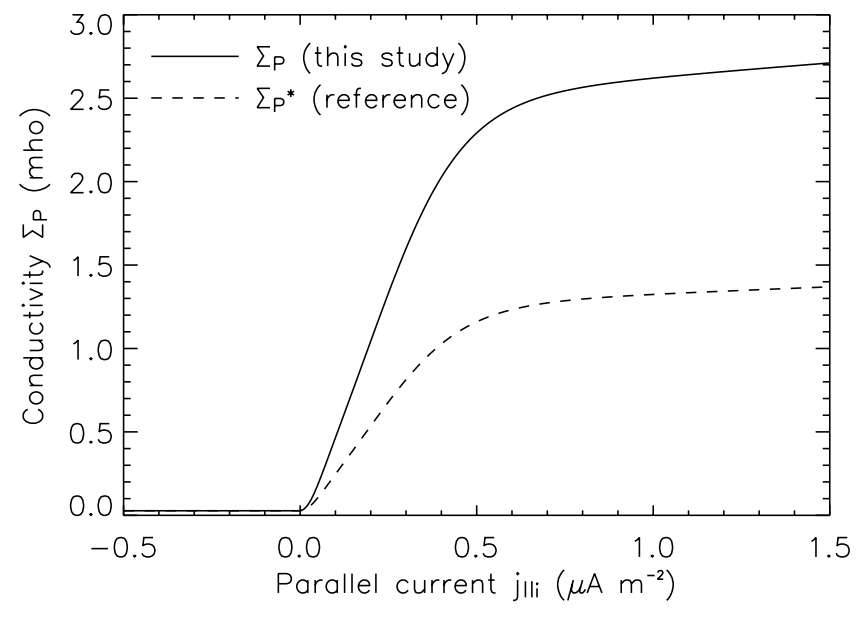

Fig. 6. Relation between ionospheric field-aligned current and true height-integrated conductivity. Solid line: true conductivity model used in this study; dashed line: effective conductivity model used by Nichols and Cowley (2004) for $\dot{M}=1000 \mathrm{~kg} \mathrm{~s}^{-1}$. Note that for negative values of the field-aligned current both models take a constant value of 0.0275 mho.

example:

$\Sigma_{P}=\int_{z 0}^{z 1} \sigma_{P} d z=\int_{z 0}^{z 1} s_{P} \rho d z=\int_{p 1}^{p 0} s_{P} g d p$

where the last step follows from hydrostatic equilibrium $d p / d z=-\rho g$, where $g$ is the acceleration due to gravity. If $g$ is constant with height (a good approximation since the vertical extent of the thermosphere is small compared to the radius of the planet) then $\Sigma_{P}$ depends only on the profile of $s_{P}$ with respect to pressure, not on the thermal structure. Similar arguments apply for $\Sigma_{H}$ and $\Omega_{T}$.

Thus employing the quantities $s_{P}$ and $s_{H}$ - which we may usefully refer to as conductivities "per unit mass" - allows us to control the height-integrated values of the conductivity and the neutral rotation velocity independent of changes in the thermal structure of the upper atmosphere. The vertical profiles of $s_{P}$ and $s_{H}$ are plotted in the right hand panel of Fig. 5. The general shape of these profiles is the same as that described for the conventional Pedersen and Hall conductivities shown in the left hand panel. However, dividing by the mass density $\rho$ (which of course decreases approximately exponentially with altitude) has the effect of respectively strengthening and weakening the high and low altitude "tails" of the conductivity distributions. Thus, there is a very rapid decrease at altitudes below the peak, but a much slower decrease with increasing altitudes, such that $s_{P}$ decreases by only one order of magnitude between the peak and the top of our model. This indicates that effects due to Joule heating and ion drag are likely to be significant in determining the energy balance and kinematics at high altitudes, even if this region is relatively insignificant in terms of the overall energy and momentum budget of the thermosphere.

The above fully specifies the shape of the vertical profile of conductivity. The magnitude of the conductivities $s_{P}$ and $s_{H}$ at a particular point in the model is determined by scaling the entire vertical profile to obtain the desired value of $\Sigma_{P}$ at that latitude, as described below. The conductivities $\sigma_{P}$ and $\sigma_{H}$ can then be calculated using the values of $\rho$ calculated from the local thermal structure of the model.

\subsection{Horizontal distribution}

The horizontal distribution of true height-integrated conductivity in regions $\mathrm{C}$ and $\mathrm{D}$ is determined directly from the magnetosphere model in terms of the field-aligned current $j_{\| i}$, as described by Eq. (17). We now expand on the precise form of this function:

$\Sigma_{P}\left(j_{\| i}\right)=\Sigma_{P 0}+\Sigma_{P j}\left(j_{\| i}\right)$

where the two components are, respectively, a "background" conductivity due to solar-produced conductivity, and an auroral enhancement that depends on $j_{\| i}$.

For the "background" conductivity, Nichols and Cowley use a value of $\Sigma_{P 0}^{*}=0.0275 \mathrm{mho}$. This is derived from the results of Hill (1980), who compared his theoretical model with the observed plasma rotation velocity (McNutt et al., 1979). This comparison fixed the ratio between the effective conductivity and the mass outflow rate. Taking $\dot{M}=1000 \mathrm{~kg} \mathrm{~s}^{-1}$, this implies $\Sigma_{P 0}^{*}=0.0275$ mho. For consistency with Nichols and Cowley we also adopt this value. However, we specify the true conductivity $\Sigma_{P 0}$. Since we do not know in advance the factor of $(1-K)$ required to convert between these two quantities, we initially assume that in the inner region of the magnetosphere to which this background conductivity applies $K \sim 0$ and set the background conductivity $\Sigma_{P 0}=0.0275$ mho.

The auroral enhancement is described using the same function as Nichols and Cowley (2004). This function was developed through detailed manipulation of the modelling results of Millward et al. (2002). In order to maximise our consistency with their model, we adopt the same form:

$$
\begin{aligned}
\Sigma_{P j}\left(j_{\| i}\right) & =0.16 j_{\| i}+\left\{2.45\left[\frac{\left(j_{\| i} / 0.075\right)^{2}}{1+\left(j_{\| i} / 0.075\right)^{2}}\right]\right. \\
& \left.\times \frac{1}{\left[1+\exp \left(-\left(j_{\| i}-0.22\right) / 0.12\right)\right]}\right\}
\end{aligned}
$$

where $\Sigma_{P}$ is in mho and $j_{\| i}$ is in $\mu \mathrm{Am}^{-2}$. Note that we use this function to specify the true conductivity, and explicitly calculate any neutral winds that may reduce the effective conductivity. Nichols and Cowley assume $K=0.5$ in order to specify an enhancement in the effective conductivity such that their $\Sigma_{P j}^{*}\left(j_{\| i}\right)=0.5 \Sigma_{P j}\left(j_{\| i}\right)$.

The solid line in Fig. 6 shows the functional form specified by Eqs. (21) and (22), taking, as discussed, a true background 
conductivity of $\Sigma_{P 0}=0.0275$. The dashed line shows the effective conductivity model of Nichols and Cowley (2004). In this case the contribution from the auroral enhancement is reduced by a factor of $K=0.5$ while the background effective conductivity is $\Sigma_{P 0}^{*}=0.0275$, as discussed above. Thus for negative values of $j_{\| i}$ our true conductivity model and the Nichols and Cowley effective conductivity model take identical constant values; for positive values of $j_{\| i}$ the enhancement to our true conductivity model is twice that to the Nichols and Cowley effective conductivity model. This latter model - for the effective conductivity - is used to calculate the reference model describe in Sect. 7.3.

For regions A and B we expect the conductivity to be enhanced over the background level, since diffuse UV and IR emission is observed in these regions, indicating some level of particle precipitation. The observed emission is rather complex (e.g. Stallard et al., 2003), but for simplicity we assume a constant conductivity across this region. We follow Cowley et al. (2005) in setting the conductivity here to $\Sigma_{P}=0.2$ mho (although, again, we note that they specified $\Sigma_{P}^{*}=0.2 \mathrm{mho}$ ). Note that at the boundary between regions A and B we expect a sheet of upward field-aligned current, which, according to Eq. (22) may enhance the conductivity (Cowley et al., 2005). For simplicity we neglect any such enhancement at this boundary; our major focus is understanding the influence of the neutral atmosphere on the middle magnetosphere (region $\mathrm{C}$ ).

\subsection{Problems with our approach}

One problem with our approach is that we use a conductivity model derived from an auroral model at all latitudes. Thus at low latitudes our "background" conductivity, which should represent conductivity due to solar-produced ionisation, has the same profile as the enhanced conductivity in the auroral zones. This, of course, is incorrect. However, if our background conductivity were of a different vertical form to the enhanced conductivity, then both the magnitude and the vertical distribution of the conductivity would vary with $j_{\| i}$. For example, if there was no precipitation, we would have a solar-produced conductivity profile, which would be likely to have a broader vertical distribution and a less intense peak; whereas in the regions where there was significant precipitation the profile would be dominated by the sharply peaked auroral profile.

The value of $\Omega_{T}$ depends on the vertical distribution of conductivity through Eq. (5) - thus if the profile depended on $j_{\| i}, \Omega_{T}$ would become a function of the field-aligned current. This considerably reduces the tractability of solving Eqs. (15-17) simultaneously. While one might envisage this dependence of $\Omega_{T}$ on $j_{\| i}$ producing some interesting effects, it seems an unnecessary complication for this initial study. Hence we tolerate some inaccuracy in the mid-latitude conductivity profile for the sake of simplicity.
Note also that the only role of the conductivity in thermospheric structure is to determine the magnitude of Joule heating and ion drag, which we expect to be most important in the polar regions where the relative velocities of plasma and neutrals are largest. In these regions the conductivity is also likely to be largely auroral in origin, so it makes sense to pick a model of the auroral ionosphere to calculate our conductivity profile. We expect pressure gradients to be a more important driver of dynamics at mid-latitudes - if so an inaccurate conductivity profile should not significantly affect our results.

A secondary source of inaccuracy at mid-latitudes is our assumption that the magnetic field is vertical at all latitudes. This is a good approximation at high latitudes and is necessary for consistency with the Nichols and Cowley (2004) model, which makes the same assumption. Of course, a vertical magnetic field is a poor assumption at mid latitudes and an extremely poor assumption at the magnetic equator where the field is horizontal. However, we find that the magnitudes of the Joule heating and ion drag terms in the equatorial region are relatively so small that we believe the impact on our results to be negligible. The exception to this is the experiment described in Sect. 8 in which the Joule heating at low latitudes is specifically enhanced; this will be discussed in more detail in Sect. 8.

\section{Coupled model}

We have described three separate component models of the magnetosphere, thermosphere and ionospheric conductivity respectively. In order to couple these models together we have ensured that shared physical quantities and assumptions are as consistent as possible:

1. We assume Jupiter to be a sphere with a radius of $R_{J}=71492 \mathrm{~km}$. Nichols and Cowley (2004) used a value of $R_{J}^{N C}=71323 \mathrm{~km}$, a difference of $\sim 0.2 \%$. We still use $R_{J}^{N C}$ to calculate the value of $F_{e}$ as a function of radial distance. Our value, $R_{J}$, is employed in all subsequent calculations, introducing very small $(\sim 0.2 \%)$ inconsistencies between our model and Nichols and Cowley (2004). Note that some ambiguity in radial distance is in any case inevitable since our ionospheric conducting layer varies in altitude according to the thermal structure, while the magnetosphere model assumes a spherical conducting layer.

2. We assume a constant vertical magnetic field $B_{i}=2 B_{J}$, where $B_{J}=426400 \mathrm{nT}$, across the whole planet. This is a simplifying assumption of the magnetosphere model in the polar regions. Identically applying this assumption to our ionosphere and thermosphere models ensures that our calculations of ion drag and subsequent angular momentum exchange with the thermosphere model are consistent with the calculated angular momentum 
transfer in the magnetosphere model. Vertical magnetic field is a poor assumption at low latitudes, but is expected to have a small effect on our results as discussed in Sect. 5.3

3. Parallel electric fields in the magnetosphere are neglected such that we can map $\Omega_{M}$ unchanged along field lines between the magnetosphere and thermosphere. This ensures that the quantity $\Omega_{M}$ discussed in the context of the magnetosphere in Sect. 3 is the same as that discussed in the context of ionospheric electric fields and currents in Sect. 2.

These assumptions allow us to alter the Nichols and Cowley (2004) model as little as possible so that we can clearly examine the influence of thermospheric dynamics on the magnetosphere.

To couple the three models together we use an iterative approach. We first solve Eqs. (15), (16) and (17) simultaneously, using the procedure outlined in Sect. 3.2.2, assuming that $\Omega_{T}=\Omega_{J}$ at all latitudes.

The resultant Pedersen conductivities $\Sigma_{P}$ are used to scale the vertical ionospheric conductivity model (Sect. 5.1) at each latitude according to the formulation discussed in Sect. 5.2. The plasma rotation velocities $\Omega_{M}$ are then used together with the ionospheric conductivities and the existing thermospheric wind speeds to calculate Joule heating and ion drag. The thermosphere model is then stepped forward in time, driven by these values of Joule heating and ion drag, which are updated each timestep as the thermospheric wind speed evolves while the values of $\Omega_{M}$ and $\Sigma_{P}$ are kept fixed. After the thermosphere model has been run for one tenth of a planetary rotation, we calculate a new magnetosphere, implying new profiles of $\Omega_{M}$ and $\Sigma_{P}$, using the values of $\Omega_{T}$ generated by the thermosphere model. We repeat this process iteratively.

Thus we run the thermosphere model continuously, calculating a new steady state magnetosphere 10 times per planetary rotation, until the thermosphere is close to a steady state. We find that this is reached reliably after 200 Jovian rotations, and adopt this runtime for all of the results shown in this study.

\section{Response of baseline model}

\subsection{General thermospheric behaviour}

The response of the baseline model is illustrated in Fig. 2 in terms of the rotation velocities of the plasma and neutrals (solid and dotted lines) and true height-integrated conductivity (dashed line). Regions A and B exhibit fixed plasma velocities as required by the model. Regions $\mathrm{C}$ and $\mathrm{D}$, for which the plasma velocity is explicitly calculated, exhibit greater structure, which will be discussed in detail below. The conductivity shows a sharp peak at the poleward edge of region $\mathrm{C}$; this is the conductivity enhancement due to the particle precipitation that forms the main auroral oval.

The neutrals show corotation of $\sim 50 \%$ in regions A and $\mathrm{B}$. Just equatorward of the main oval, in region $\mathrm{C}$, is a region of super-corotation. Similar super-corotation was observed by Smith and Aylward (2008) in the case of Saturn. Further towards the equator the neutral velocity returns to the planetary rotation velocity.

A more detailed view of the thermospheric dynamics is shown in Fig. 7, which shows the temperatures (top), winds (middle) and Pedersen conductivities, Joule heating and ion drag energy (bottom). The temperature structure is, in outline, identical to that described for Saturn by Smith et al. (2007), with a hotspot at the pole and rather inefficient redistribution of thermal energy to regions equatorward of the main oval. While the polar temperatures peak at $\sim 700 \mathrm{~K}$, which approaches the $\mathrm{H}_{3}^{+}$temperature measurements in this region (Lam et al., 1997; Stallard et al., 2002), the low latitudes retain cool temperatures close to those generated by absorption of sunlight.

The winds also exhibit similar structures. The zonal winds exhibit a single broad sub-corotating jet in regions A and B; this terminates rather sharply at the boundary with region $\mathrm{C}$. This is associated with the sudden change in the plasma velocity in the region of the main oval. Most importantly, the poleward flow at low altitudes that cools mid latitudes (Smith et al., 2007) is clearly present, indicating that this dynamical process is a feature common to Jupiter and Saturn.

It is worth briefly summarising the analysis of this poleward flow presented by Smith et al. (2007). The westward winds generated by ion drag are acted on by Coriolis forces which generate strong poleward flows throughout the region coupled to sub-corotating plasma. At the boundary between corotating and sub-corotating plasma - in this case corresponding approximately to the location of the main auroral oval - there is a divergence in this poleward flow which drives upwelling from lower altitudes. The upwelling gas cools adiabatically, producing a region just equatorward of the main auroral oval that is cooler than the lower boundary temperature. The poleward pressure gradient on the equatorward edge of this cool region drives gas towards the pole. Since there is negligible westward ion drag in this region, Coriolis forces drive this gas into super-corotation. One overall effect of sub-corotational ion drag at the pole is therefore a cool, super-corotating region just equatorward of the main oval.

The distribution of energy inputs is also interesting. Regions $\mathrm{A}$ and $\mathrm{B}$ exhibit significant Joule heating and ion drag powers at low altitudes. This is simply due to the action of the magnetospheric frictional drag in the region that has the highest conductivity. At high altitudes there are areas of negative ion drag energy in both of regions B and C. Both of these regions of ion drag represent extraction of kinetic energy stored in the thermally-driven high-altitude winds that are sub-corotating relative to the plasma. Some of the K.E. 
from these regions is thermalised - generating a region of Joule heating at high altitudes in region $\mathrm{C}$ - and some is redistributed, either to the magnetosphere or to lower altitudes.

The high altitude region of Joule heating in region $\mathrm{C}$ is also interesting because it is partly a conductivity enhancement effect. Thermally-driven winds blowing through the main auroral oval encounter resistance from the enhanced conductivity in this region, which slows the winds and extracts kinetic energy as heat. Were the conductivity not enhanced, the Joule heating in this region would be relatively unimportant.

Indeed, the most interesting aspect of the energy input distributions shown is that the Joule heating due to the main oval - i.e. due to the middle magnetosphere-thermosphere coupling currents - is much less significant than that due to the greater sub-corotation rates over a much greater area that are present inside the polar cap. This indicates that the main oval itself may be relatively unimportant in terms of the thermal structure.

\subsection{Momentum balance}

We now summarise the height-integrated momentum balance of the thermosphere. We calculate a height-averaged velocity which represents the total momentum per unit mass of a column of thermosphere at a given location:

$\bar{u}_{\phi}=\frac{\int \rho(z) u_{\phi}(z) d z}{\int \rho(z) d z}$

It should be emphasised that this weighted height-average is of a different nature to the weighted height-average used to calculate $\Omega_{T}$ (Eq. 5). In this case, we are weighting the thermospheric velocity by mass in order to summarise the overall momentum budget of the thermosphere. In the case of $\Omega_{T}$ we weight the thermospheric velocities according to the Pedersen and Hall conductivities in order to summarise the specific process of momentum exchange with the magnetosphere.

We can also calculate height-averaged momentum terms, analagous to $\bar{u}_{\phi}$, which represent the total column rate of change of momentum per unit mass due to the various terms in the momentum equation. Figure 8 shows the plasma velocity $v_{\phi}$ and height-averaged neutral velocity $\bar{u}_{\phi}$ in the upper panel and the corresponding height-averaged zonal momentum terms in the lower panel. The upper panel has been shaded to give an impression of the true height-integrated Pedersen conductivity at each co-latitude. Thus the darkest shaded region corresponds to the conductivity enhancement due to the main auroral oval. We group together the momentum terms into advection (horizontal and vertical) viscous drag (horizontal and vertical), inertial terms (Coriolis and curvature) and ion drag.

This analysis shows that the behaviour is very similar to that described for Saturn by Smith and Aylward (2008). Viscous drag is insignificant. Everywhere ion drag acts to increase the westward velocity and this is opposed almost everywhere by the Coriolis force. Across most of the

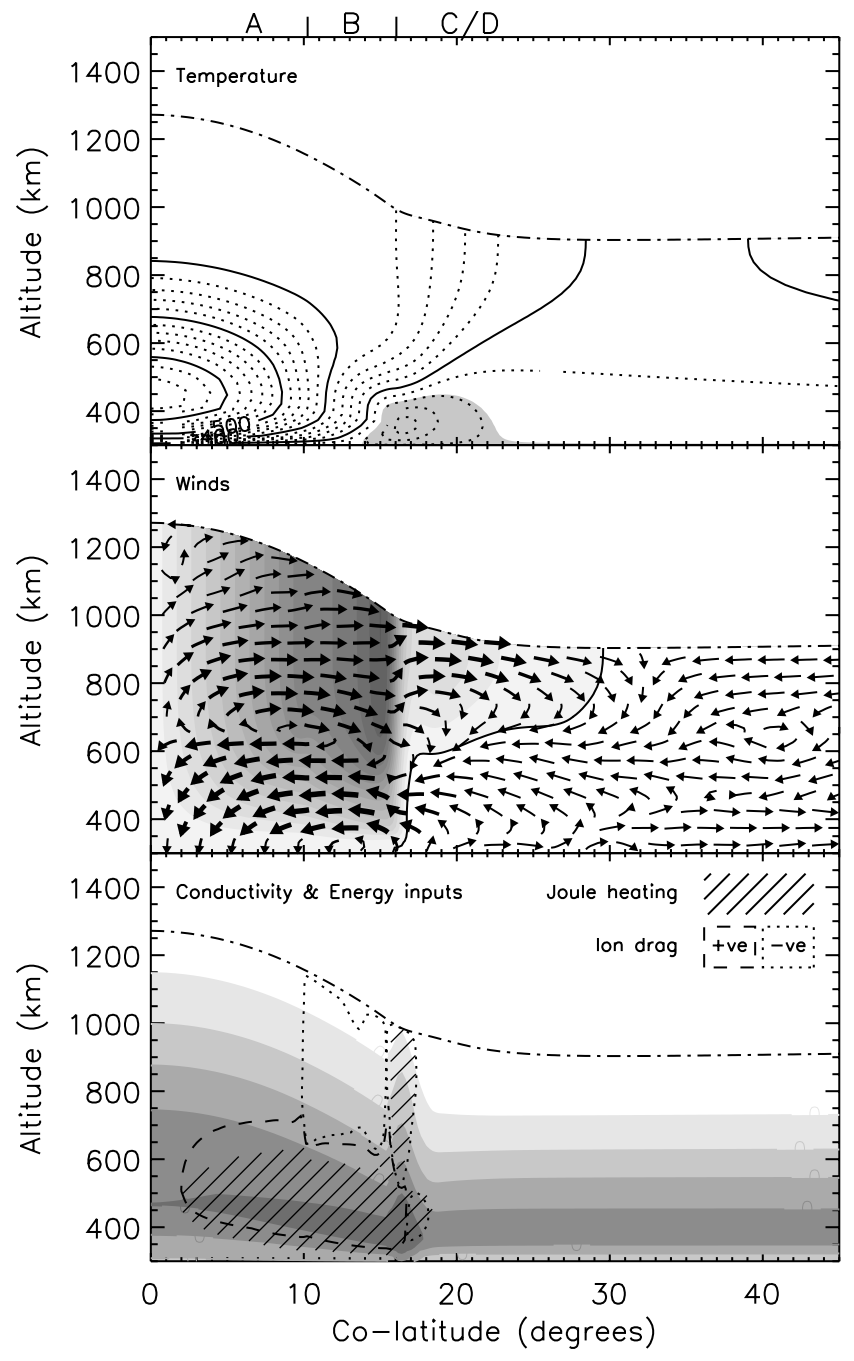

Fig. 7. Temperatures, winds and energy inputs for our baseline model. Top: temperatures in $\mathrm{K}$ are shown by the contours. Solid contours are plotted every $100 \mathrm{~K}$ and dotted contours at $20 \mathrm{~K}$ intervals inbetween. The highest temperature solid contour, on the left of the plot, is at $600 \mathrm{~K}$; the solid contour at the far right hand side is at $300 \mathrm{~K}$. Grey shading shows areas that are cooler than the lower boundary temperature of $262 \mathrm{~K}$. Middle: winds. Grey shading shows westward winds, the darker shading indicating greater speeds. Eastward winds are in general of much smaller magnitude; these regions are not shaded. The solid contour represents zero zonal wind speed, thus dividing regions of westward and eastward winds. Arrows show the combined meridional and vertical circulation. The thickness of the arrows is indicative of the combined meridional and vertical wind speed. Bottom: Conductivity distribution and energy inputs. The grey shading shows the distribution of Pedersen conductivity $\sigma_{P}$. The darkest region represents conductivities greater than $10^{-6} \mathrm{mho} / \mathrm{m}$; the next darkest region conductivities greater than $10^{-7} \mathrm{mho} / \mathrm{m}$ and so on until the unshaded region represents conductivities less than $10^{-10} \mathrm{mho} / \mathrm{m}$. Diagonal hatching indicates Joule heating in excess of $2 \mathrm{~W} / \mathrm{kg}$. Dashed contours enclose regions in which ion drag inputs kinetic energy at a rate in excess of $2 \mathrm{~W} / \mathrm{kg}$; dotted contours enclose regions in which ion drag extracts kinetic energy at a rate in excess of $2 \mathrm{~W} / \mathrm{kg}$. 

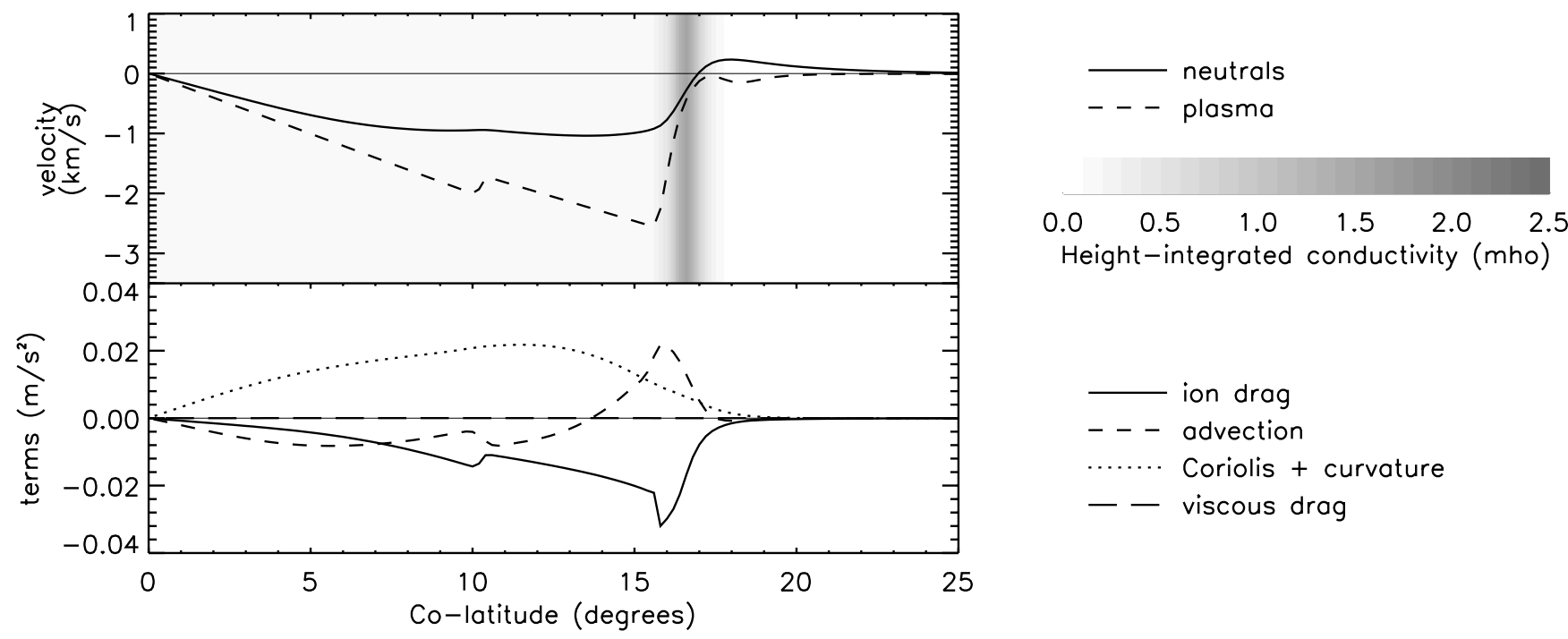

Fig. 8. Height-averaged zonal velocities and momentum terms for our baseline model. Upper plot: height-integrated zonal neutral velocity (solid line) and zonal plasma velocity (dashed line). Shading represents the value of the true height-integrated Pedersen conductivity. Lower plot: height-averaged zonal momentum terms, grouped into ion drag (solid line), advection (short dashed line), Coriolis and curvature (dotted line) and viscous drag (long dashed line). Note that the latter line, representing viscous drag, is almost contiguous with the $\mathrm{x}$-axis for the entire range shown.

shown region advection acts in the same direction as ion drag, i.e. towards sub-corotation. This is due to the subcorotational zonal velocity decreasing towards the pole, the prevailing meridional wind being poleward. In the region of the main oval advection becomes important in supporting the flow against sub-corotation, since in this region the sub-corotational zonal velocity increases towards the pole. Gas that is advected poleward across the main oval supports the flow against sub-corotation because it has arrived from a region where both the plasma and neutrals almost corotate. There is a lag before it acquires the greater sub-corotation typical of the polar regions A and B.

Just equatorward of the main oval Coriolis forces again become more important than advection, and, as at Saturn, it is this that generates the small region of super-corotation in the neutral velocity profile.

\subsection{Response of magnetosphere}

Whereas the thermospheric response is clearly similar to that calculated for Saturn (Smith et al., 2007; Smith and Aylward, 2008), the use of a physical model of the middle magnetosphere allows us to directly assess the influence of the thermospheric winds on the plasma flows in this region. In the following, we will always plot our results alongside a reference model corresponding to the assumptions of Nichols and Cowley (2004). Specifically, our reference model corresponds to the results shown in Fig. 15 of their paper, for a mass-loading rate $\dot{M}=1000 \mathrm{~kg} \mathrm{~s}^{-1}$. The calculation of the reference model was used to validate our method of obtaining solutions for the middle magnetosphere. In general we plot the results of the reference model as a dashed line and those of our full model as a solid line.

The reference model is calculated using an almost identical model of the true ionospheric conductivity, but with the neutral rotation velocity calculated assuming that $K=0.5$. The only small difference between the conductivity model used for the reference model and that used for our full model runs, is that the reference model assumes an effective background conductivity of $\Sigma_{P}^{*}=0.0275 \mathrm{mho}\left(\Sigma_{P}=0.055 \mathrm{mho}\right.$, $K=0.5$ ) whereas the full model uses a true background conductivity of $\Sigma_{P}=0.0275 \mathrm{mho}$, the effective conductivity $\Sigma_{P}^{*}$ then following from the value of $K$ that is implied by the thermosphere model (see discussion in Sect. 5). We will discuss the effect of our assumed background conductivity further below.

Figure 9 shows the standard format in which we present our results. The results are plotted as a function of radial distance in the magnetosphere. Our model extends from $4 R_{J}$ to $100 R_{J}$; within $4 R_{J}$ we assume $\Omega_{M}=\Omega_{T}$. The left hand column represents parameters associated with the rotation velocities of the plasma and neutrals, and the right hand column parameters associated with currents and conductivities.

Figure 9a is the effective thermospheric rotation velocity $\left(\Omega_{T}\right)$. The dashed line shows the thermospheric rotation velocity implied by the reference model. Since the reference model assumes $K=0.5$, this is calculated by halving the plasma corotation lag. Figure $9 \mathrm{~b}$ shows the plasma angular 

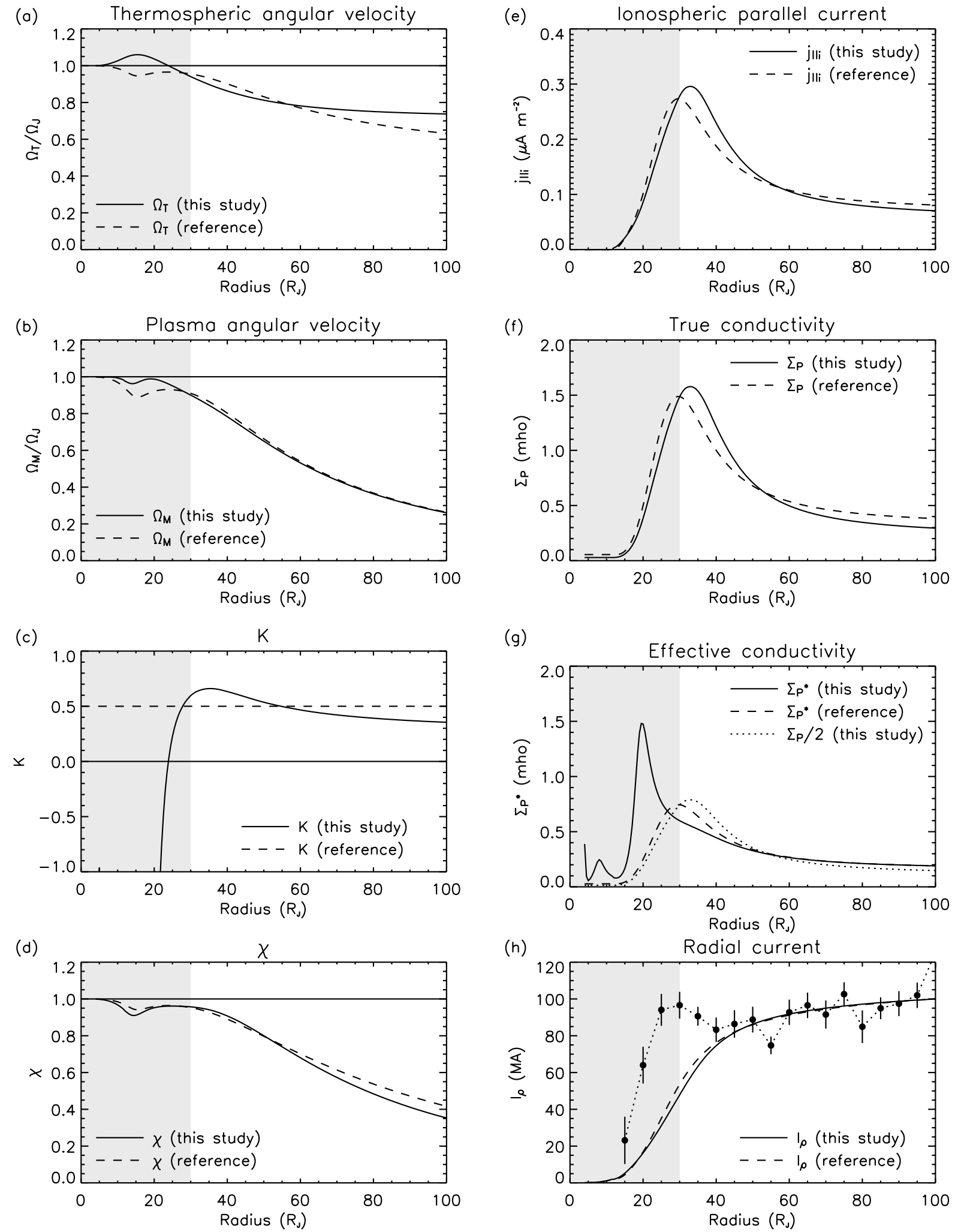

Fig. 9. Magnetospheric parameters for our baseline model. Solid lines: full model. Dashed lines: reference model. (a) $\Omega_{T}$, (b) $\Omega_{M}$, (c) $K$, (d) $\chi$, (e) $j_{\| i}$, (f) $\Sigma_{P}$, (g) $\Sigma_{P}^{*}$ (dotted line shows $\Sigma_{P} / 2$ for the full model), (h) $I_{\rho}$ (dotted line shows values deduced from the data of Khurana, 2001). 
velocity $\Omega_{M}$. Again, the reference model is represented by a dashed line. Figure 9c shows our calculated value of $K$ mapped into the magnetosphere. The horizontal dashed line represents the reference model assumption that $K=0.5$. Figure $9 \mathrm{~d}$ shows the ratio $\chi=\Omega_{M} / \Omega_{T}$. This parameter is useful in comparing the relative plasma and neutral rotation velocities; it was also used by Smith and Aylward (2008) to specify the plasma rotation velocities as a function of the neutral rotation velocities.

Figure $9 \mathrm{e}-\mathrm{h}$ shows, respectively, the field-aligned current in the ionosphere (mapped to the magnetosphere), the resulting true height-integrated conductivity, the effective heightintegrated conductivity, and the radial current in the magnetosphere. Figure 9g also includes a dotted line which indicates the effective conductivity that we would calculate if we halved our true conductivity calculated with the full model. This gives an indication of the features in the effective conductivity that are due to structure in $K$ rather than structure in the true conductivity.

Finally, the dotted line in Fig. 9h shows values of the radial current deduced from Galileo magnetometer data (Khurana, 2001). These are the same values shown by Nichols and Cowley (2004); for further details of the origin of these values, the reader is referred to both of these papers.

Initial inspection of Fig. 9 indicates that the introduction of coupling to the neutrals has only a small effect on the majority of the magnetospheric parameters. In particular, the plasma angular velocities calculated using the full model are effectively identical to the reference model beyond $\sim 30 R_{J}$. At smaller radial distances our plasma angular velocity is considerably greater than that in the reference model, lying much closer to corotation. These two regimes divide the model naturally into "outer" and "inner" regions, respectively. The region within $30 R_{J}$ is shaded grey to represent this division.

Looking first at the "outer" region, beyond $30 R_{J}$, we can see that although the plasma angular velocity is almost unchanged, each of the other parameters differs, at least marginally, from the reference model. These differences can be traced to the differing behaviour of the neutral atmosphere, which rotates slightly more slowly than in the reference model between $30 R_{J}$ and $55 R_{J}$, and then slightly more quickly beyond $55 R_{J}$. This slight difference in the neutral rotation velocities is because our model does not generate radially constant profiles of $K$, and it is thus unsurprising that we do not exactly reproduce the reference model. However, the model does produce values of $K$ in the range $0.35-0.7$ throughout the whole outer region. This suggests that the assumption $K \sim 0.5$ is reasonable in this part of the magnetosphere. This is presumably because, as is clear from Fig. 3, most of the magnetosphere beyond $20 R_{J}$ maps to a very confined range of latitudes in the thermosphere, implying a fairly homogeneous behaviour. It must be emphasised, though, that although a numerical value of $K \sim 0.5$ is a good match in this region, this does not mean that the physical meaning of $K$ implied by Huang and Hill (1989) is valid (see Sect. 2.5).

In the outer region our currents are distributed very slightly differently from the reference model. The auroral oval maps to a slightly larger radius, and consequently the radial current is concentrated very slightly more in the outer regions of the magnetosphere. However, these small changes do not seem particularly significant, and are simply minor consequences of $K$ not being exactly equal to 0.5 .

The inner region is considerably more interesting. To aid our discussion of this region, Fig. 10 shows the same parameters as Fig. 9, but across the range $0-40 R_{J}$ only. This region maps to latitudes just equatorward of the main auroral oval, which exhibit super-corotation via the mechanism described in Paper 1. Super-corotation was not envisaged when the parameter $K$ and the concept of effective conductivity were introduced (Huang and Hill, 1989), so the consequences of this behaviour are somewhat peculiar, as anticipated in Sect. 2. Firstly $K$ becomes strongly negative, indicating that the neutral atmosphere is deviating from corotation in the opposite sense to the plasma, and to a much greater degree - i.e. it is super-corotating much more than the plasma is sub-corotating. There are two deep troughs in $K$ in this region, each of which corresponds to peaks in the plasma angular velocity. As the plasma angular velocity approaches corotation, the factor $\left(\Omega_{J}-\Omega_{M}\right)$ in the formula for $K$ (Eq. 7) approaches zero, while the left hand side is negative. This generates strongly negative values of $K$, whose only physical significance is that the plasma angular velocity is close to corotation. This structure in $K$ is similar to that calculated for Saturn by Smith and Aylward (2008), since it is ultimately a consequence of $\Omega_{T}$ behaving partly independently of the profile of $\Omega_{M}$, behaviour that the constant $K$ model cannot account for.

The effect of these negative values of $K$ on the effective conductivity is to enhance it significantly, since the factor $(1-K)$ becomes strongly positive. This explains the two peaks in Fig. 9g. Since the effective conductivity represents the effective ability of the thermosphere to enforce corotation, it is not surprising that when the thermosphere supercorotates it is able to enforce corotation more effectively.

This interpretation is borne out by the behaviour of the sub-corotation parameter $\chi$, which lies very close to that calculated from the reference model. This indicates that the sub-corotation of the plasma relative to the neutrals is relatively model-independent: introducing internal dynamics of the thermosphere merely shifts the absolute value of the plasma angular velocity.

The negative $K$ values also enhance our effective conductivity in the inner region above that in the reference model, even though we assume a lower true background conductivity. The effective conductivity is the empirically constrained parameter in this region, since this almost directly determines the plasma rotation velocity (McNutt et al., 1979; Hill, 1980), 

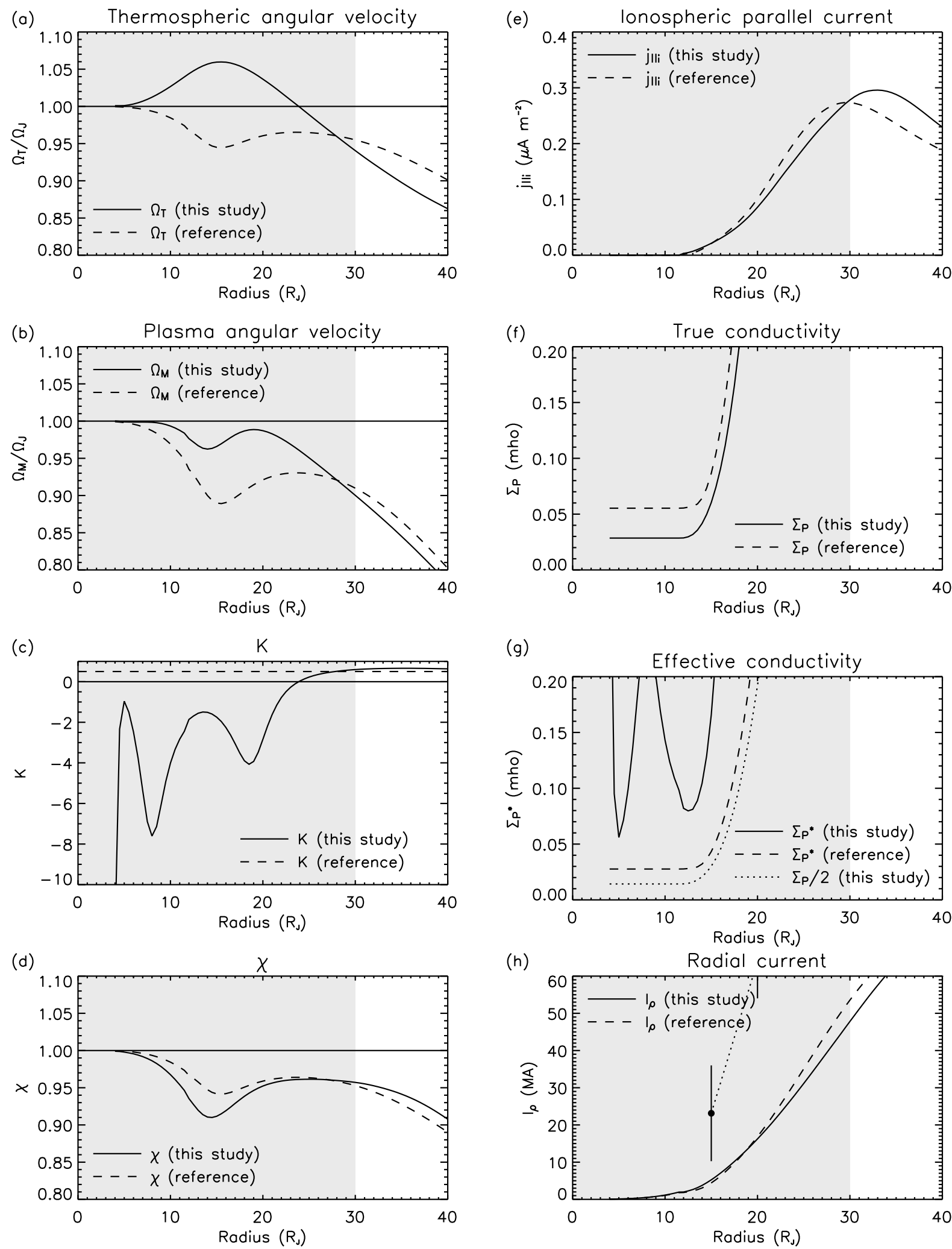

Fig. 10. Magnetospheric parameters for the baseline model in the inner region, in the same format as Fig. 9. 
and we are currently overestimating both. This suggests that we may require a still lower true background conductivity.

To investigate this, the model has been run with the true background conductivity reduced by a factor of two to $\Sigma_{P 0}=0.01375 \mathrm{mho}$, the results of which are shown, for the inner region, in Fig. 11. This reduction in the conductivity has an almost imperceptible effect on the super-corotating thermospheric angular velocity profile in the inner region. This is unsurprising, since the very fact that the thermosphere super-corotates in this region shows that its behaviour is not directly governed by sub-corotational ion drag. As discussed in Sect. 7, the super-corotation is generated by pressure gradients equatorward of the region of greatest plasma subcorotation. It thus seems that the degree of super-corotation in the part of the thermosphere connected to the inner magnetosphere is governed not by the background conductivity in that region but by the elevated conductivities and plasma sub-corotation in the main oval and polar cap, which are connected to the middle and outer magnetospheres. It is interesting that the sub-corotation of the outer regions of the magnetosphere should, indirectly, be responsible for supporting the corotation and even super-corotation of the inner regions.

Although the thermospheric rotation velocity is almost unaffected by the reduced background conductivity, the plasma corotation lag within $\sim 20 R_{J}$ increases by approximately a factor of two. Thus the background conductivity remains directly important in this region in terms of determining the degree of corotation of the magnetosphere. Within $\sim 13 R_{J}$ our model now reproduces the reference model almost exactly, and this corresponds to our effective conductivity matching the reference model well. Thus, by appropriately choosing the background conductivity, we can reproduce the empirical effective conductivity in the inner region.

However, we will see in the experiments that follow that the effective conductivity of the inner region is also sensitive to other parameters. Therefore no single value of the true conductivity can definitively give the correct effective conductivity under all conditions. Given this complexity we will use our original assumed background conductivity of $\Sigma_{P 0}=0.0275$ mho for the remainder of the study.

\section{Effect of fluctuating electric fields}

The above establishes that the basic behaviour of the middle magnetosphere and auroral oval is relatively unaffected by the winds driven by our baseline thermosphere model. Other than the super-corotation in the inner magnetosphere, our results are consistent with those of previous studies.

We now examine the sensitivity of this baseline response to the parameters that define the thermosphere model, in particular the thermospheric temperature. We introduce extra Joule heating into the thermosphere model, such as may be induced by small-scale fluctuations in the electric field, a possibility discussed by Smith et al. (2005a). Our approach is to assume an r.m.s. fluctuation of $\Delta E$ in the electric field across a range of latitudes. This introduces an extra component of Joule heating $q_{J f}$ :

$q_{J f}=\sigma_{P} \Delta E^{2}$

Note that we do not explicitly specify a fluctuating electric field. We specify the r.m.s. fluctuation $\Delta E$ and then use the formula above to estimate the resultant heating.

We study two cases: firstly, that the fluctuations arise in the solar wind, and thus affect regions A and B of our model; secondly, that the fluctuations arise in the inner magnetosphere, and affect low latitudes connected to region D. We have found that introducing fluctuations in region $\mathrm{C}$ produces very similar results to those due to fluctuations in regions $\mathrm{A}$ and $\mathrm{B}$, so for brevity these results are omitted from this report.

We show the results of two runs, both with r.m.s. fluctuations in the electric field of $\Delta E=1.0 \mathrm{~V} / \mathrm{m}$. This value is not used with any empirical justification, other than that values an order of magnitude lower than this have a negligible effect on the thermospheric temperature and values an order of magnitude higher produce temperatures far in excess of those that are observed. Thus if electric field fluctuations are responsible for the high thermospheric temperatures, they must be close to this value.

In the first run, the fluctuations are applied to regions $\mathrm{A}$ and $\mathrm{B}$, modelling a large increase in the energy entering the polar cap. In the second run, the fluctuations are applied to all regions equatorward of $20^{\circ}$ colatitude, modelling a hypothetical low-latitude energy source. Both of these distributions can also be considered as generic energy sources representing some other process that we do not identify: our primary objective is to understand how the winds driven by such an energy source may affect the rotational coupling of the thermosphere and magnetosphere.

In the case of the second run, modelling low-latitude heating, the inaccuracy of our ionosphere model at equatorial latitudes - assuming a vertical magnetic field and employing an auroral conductivity model - is a problem. However, the assumption of a vertical magnetic field is not a significant problem in the case of Joule heating due to rapidly fluctuating electric fields, since they will have a local heating effect independent of magnetic field direction. The use of an auroral conductivity model is more of a problem. However, it is very difficult to estimate how inaccurate our auroral conductivity profile is at the equator. The results from this run close to the equator should therefore not be overinterpreted.

\subsection{Polar cap electric field fluctuations}

\subsubsection{Thermospheric response}

We now show in Fig. 12 the equivalent plot to Fig. 7 for the run with additional polar cap heating. The extra Joule heating term due to fluctuations is shown by horizontal hatching, 

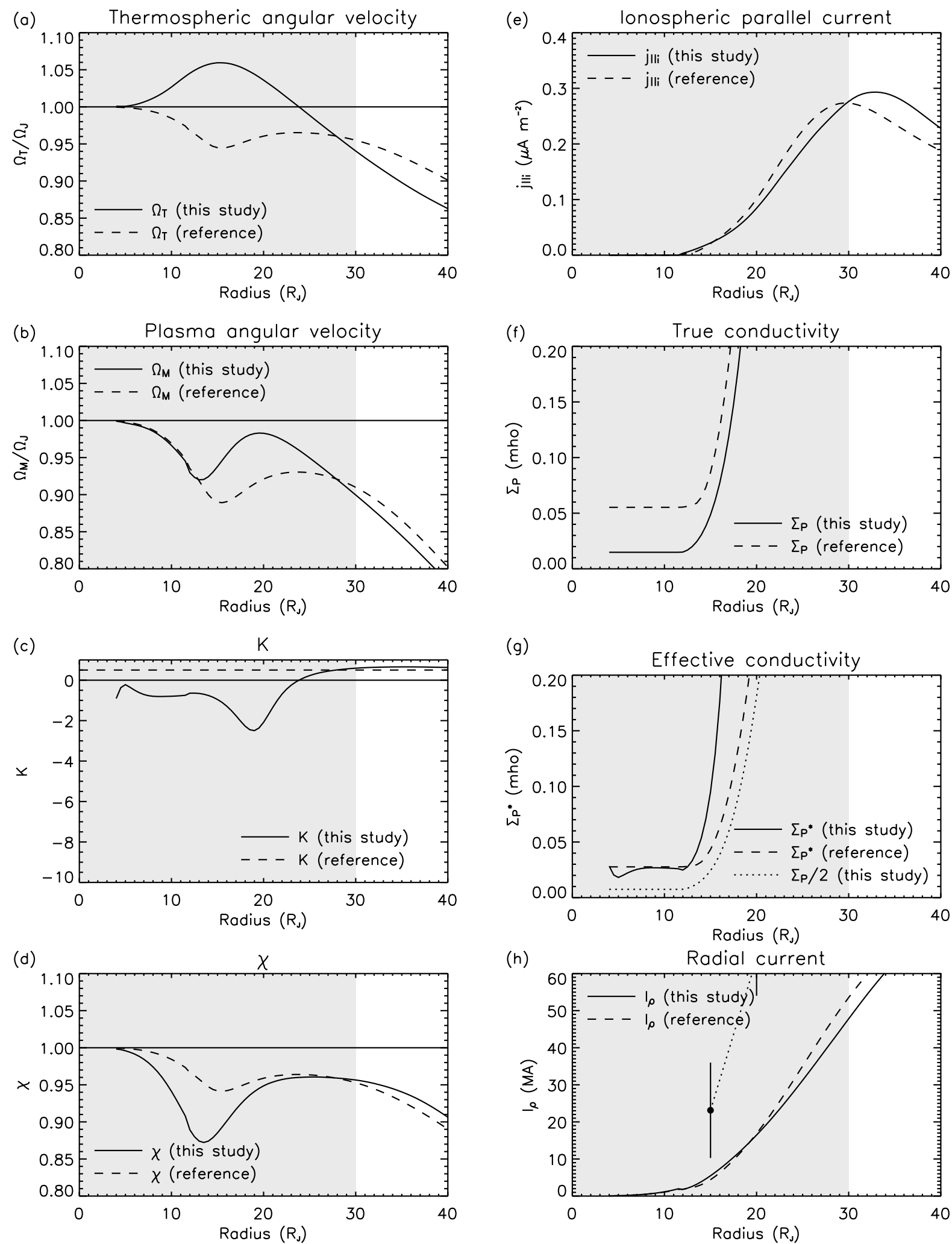

Fig. 11. Magnetospheric parameters for the inner region, with the true background conductivity reduced to $\Sigma_{P 0}=0.01375 \mathrm{mho}$. 


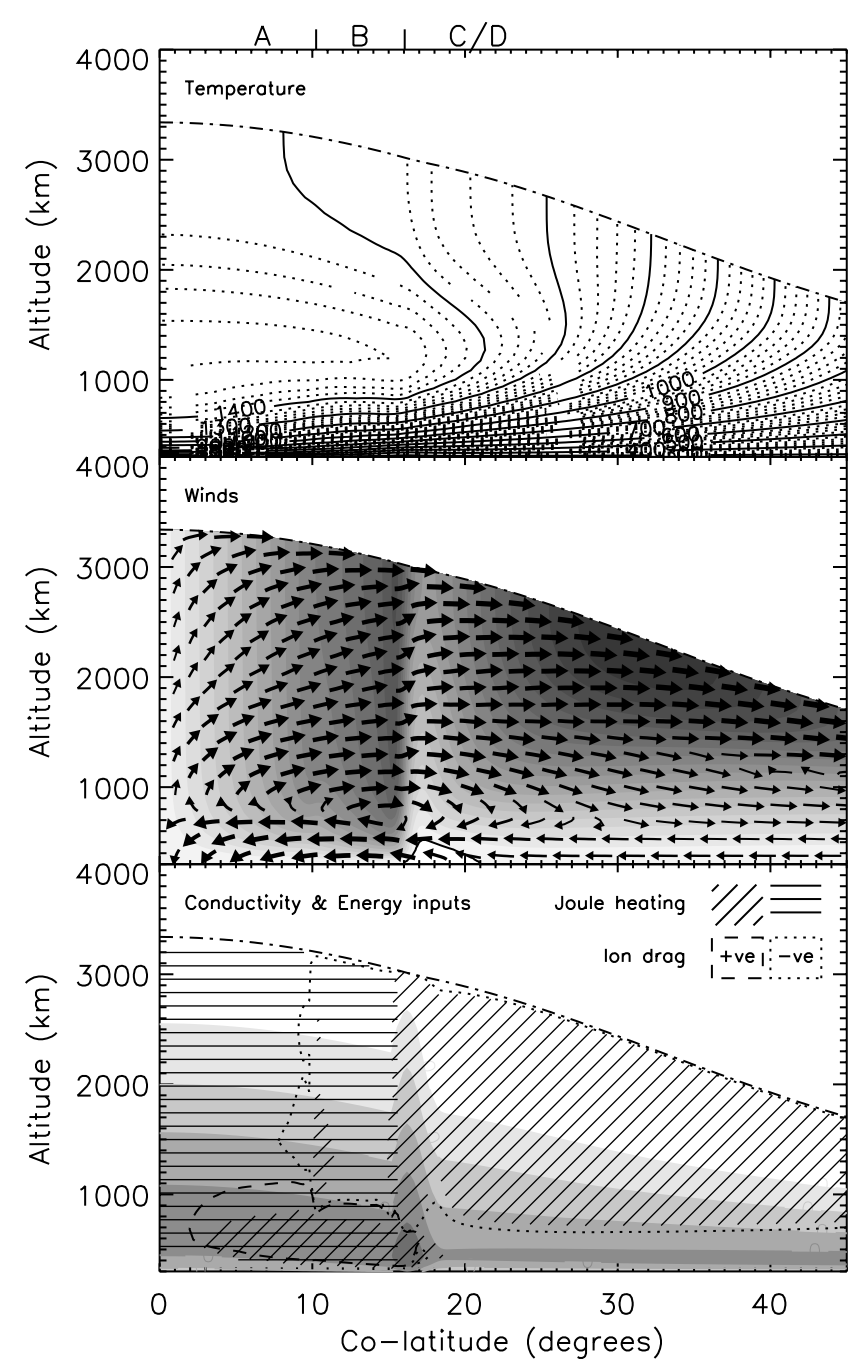

Fig. 12. Temperatures, winds and energy inputs for r.m.s. fluctuations of $1.0 \mathrm{~V} / \mathrm{m}$ applied to regions A and B only, in the same format as Fig. 7. In the bottom plot, regions in which the fluctuation heating term is greater than $2 \mathrm{~W} / \mathrm{kg}$ are shown by horizontal hatching.

while mean field Joule heating is still represented by diagonal hatching. Again, the energy input due to each process inside the corresponding hatched regions is greater than $2 \mathrm{~W} / \mathrm{kg}$.

The heating due to the electric field fluctuations has a significant effect on the thermal structure, with temperatures in the polar cap of almost $1500 \mathrm{~K}$. These temperatures are somewhat greater than the range $700-1250 \mathrm{~K}$ determined from spectroscopy of $\mathrm{H}_{3}^{+}$(Lam et al., 1997; Stallard et al., 2002). It is clear that within our current model adding any further fluctuation heating would lead to an even greater inconsistency between our predicted temperatures and the data.

The thermally driven winds now dominate the high altitude behaviour. There is significant Joule heating at all latitudes equatorward of the main oval as ion drag thermalises these winds. The Joule heating and ion drag energy in the polar cap are confined to very low latitudes, below the main conductivity peak, and in this region there is still a poleward flow. However, the behaviour in this region is now apparently of minimal importance to the overall thermal structure.

At $45^{\circ}$ colatitude the exospheric temperature is raised to $\sim 900 \mathrm{~K}$ by meridional advection. However, at the equator (not shown) the exospheric temperature is only $400 \mathrm{~K}$. This falls short by a considerable margin of the $\sim 900 \mathrm{~K}$ determined by the Galileo probe (Seiff et al., 1998). Thus our initial conclusion is that neither mean field Joule heating/ion drag nor fluctuation heating in the polar cap can account for the elevated low latitude temperature while remaining consistent with the temperatures at high latitudes.

\subsubsection{Magnetospheric effects}

We now consider the effect of these thermospheric wind systems on the behaviour of the middle magnetosphere (Fig. 13). Both the thermosphere and magnetosphere experience reduced rotation velocities. This is consistent with the increasing importance of the high altitude thermally driven winds, which are exclusively sub-corotational. Adding heating in the polar regions almost entirely removes the mechanism that generates super-corotation in the region mapping to the inner magnetosphere, by imposing an equatorward pressure gradient that is much greater than any poleward pressure gradient generated through the mechanism discussed in Sect. 7. Note that the parameter $\chi$ remains close to the reference value for all these runs, consistent with our earlier observation that most of the structure in the plasma velocity is simply due to the magnetosphere responding almost identically to a different neutral velocity profile.

The magnetosphere-thermosphere coupling currents are also affected. The peak of field-aligned current related to the aurora becomes broader and less intense and is shifted to slightly greater radial distances in the magnetosphere. At the planet, an observable consequence of this change would be a broadening and weakening of the main auroral oval and a slight poleward shift. As a consequence of this broadening of the peak in the field-aligned current the radial current rises more slowly with radial distance. The results of Nichols and Cowley (2004) imply that the region of field-aligned current behaves in this manner if the mass outflow rate of iogenic plasma $\dot{M}$ is decreased. We can interpret this in terms of a decrease in the intensity of the current systems required to enforce corotation when the mass outflow rate is lower. In our situation, the entire thermosphere is sub-corotating considerably due to its internal dynamics. Thus the magnetosphere "sees" a planet that is (differentially) rotating much more slowly than the full rotation rate of Jupiter. This produces an effect similar to a decrease in the mass outflow rate, since the amount of angular momentum required for "corotation" with the sub-corotating thermosphere is of course much less. 

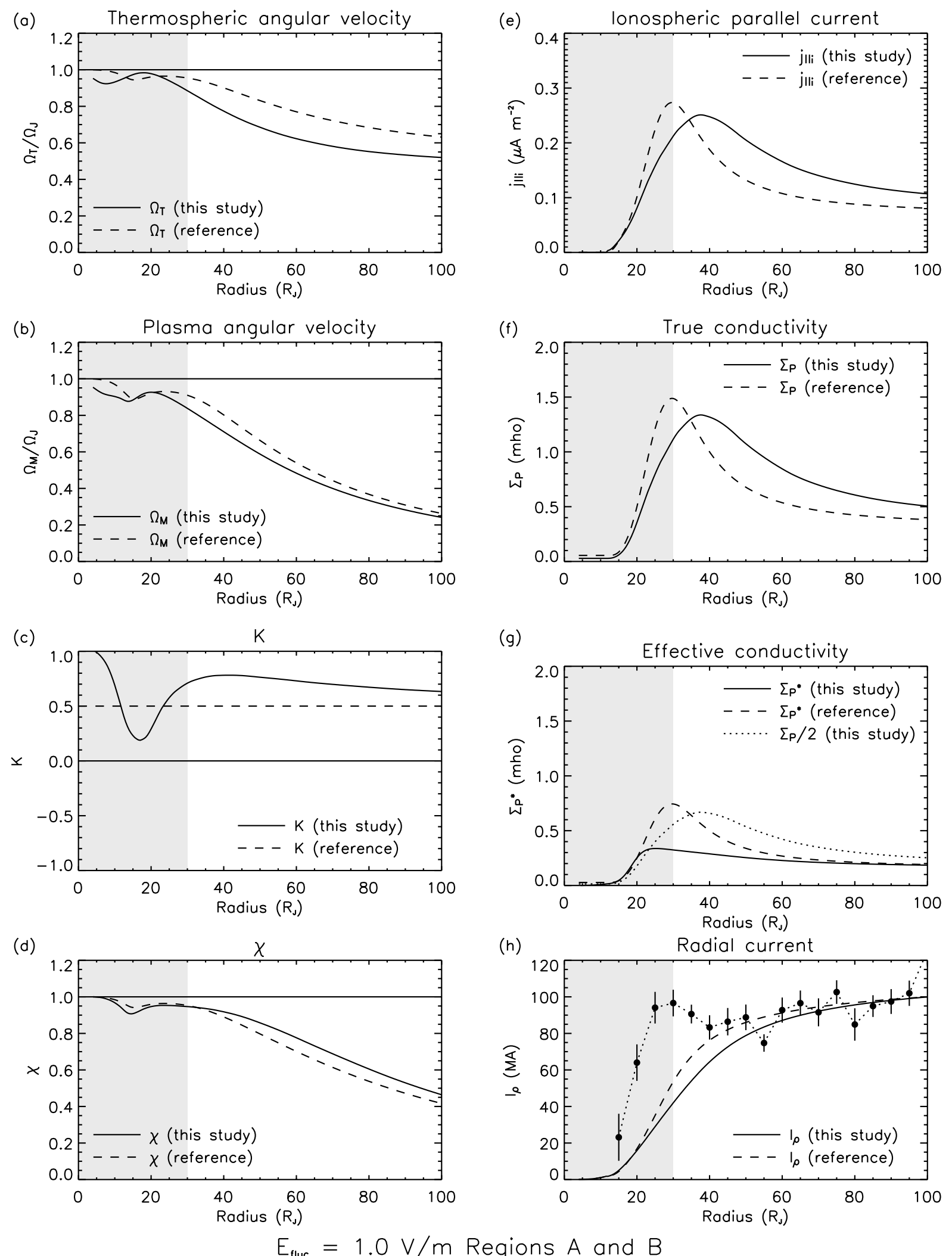

$$
E_{\text {fluc }}=1.0 \mathrm{~V} / \mathrm{m} \text { Regions } A \text { and } B
$$

Fig. 13. Parameters for r.m.s. fluctuations of $1.0 \mathrm{~V} / \mathrm{m}$ in regions $\mathrm{A}$ and B, in the same format as Fig. 9. 


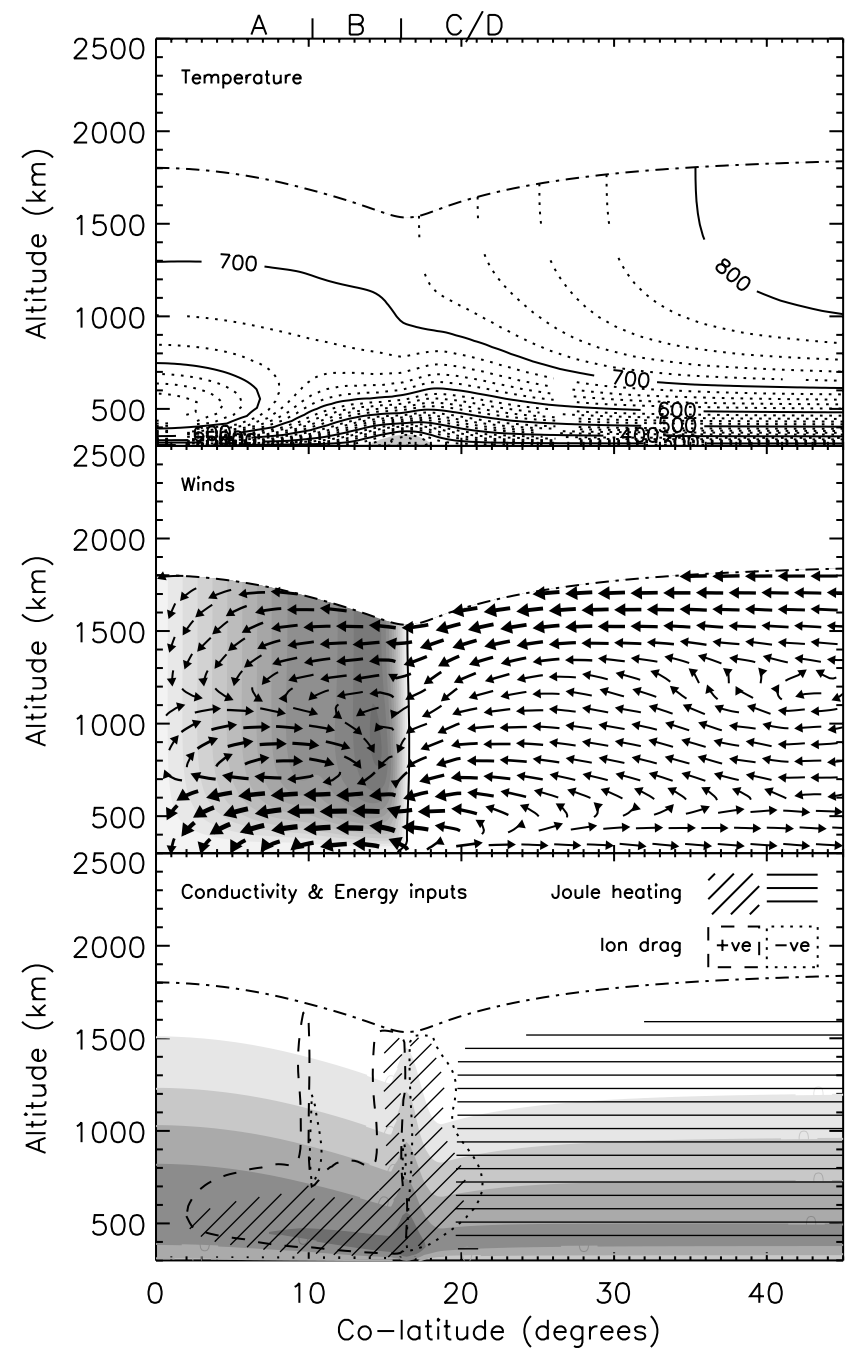

Fig. 14. Temperatures and winds for r.m.s. fluctuations of $1.0 \mathrm{~V} / \mathrm{m}$ applied equatorward of $20^{\circ}$ colatitude, in the same format as Fig. 12.

While this interpretation provides an explanation for the observed change in the coupling currents, the change itself does nothing to improve consistency with the available data. Nichols and Cowley (2004) attempted to improve the fit between their model and the data of Khurana (2001) (the dotted line in plot (h) in the figures) by increasing the mass outflow rate, thus intensifying and pushing inwards the peak in the field-aligned current. This in turn pushed inwards the rise in the radial current that occurs in the range $20-40 R_{J}$ in the reference model. In the data this rise occurs rather steeply at $\sim 20 R_{J}$. The influence of the thermally driven winds is to delay this rise in the radial current, thus making the mismatch between the data and the model worse. We can view this as indirect evidence against the hypothesis that redistribution of polar heating is responsible for the high equatorial thermospheric temperatures.
This discussion demonstrates the usefulness of a fully coupled model. We have made a modification not to the magnetosphere model but to the energy inputs to the thermosphere model. This change has had a knock-on effect in the magnetosphere, which has altered two observables: the intensity, width and location of the main auroral oval and the profile of radial current in the magnetosphere. The output of our model can be compared to these observables, setting constraints on the thermospheric heating. The coupled model thus broadens the data set that we can call upon to study thermospheric heating, by requiring that both the thermospheric and magnetospheric parameters predicted by the model match the data well.

\subsection{Equatorial electric field fluctuations}

\subsubsection{Thermospheric response}

Figure 14 shows temperatures and winds for the run with electric field fluctuations applied equatorward of $20^{\circ}$ colatitude, in the same format as Fig. 12. The overall flow is now poleward, driven by the pressure gradient generated by the equatorial heating. There is now a clear boundary between the sub-corotating winds in regions $\mathrm{A}$ and $\mathrm{B}$ and the supercorotating winds in regions $\mathrm{C}$ and $\mathrm{D}$. This leads to a similar distribution of Joule heating and ion drag in regions $\mathrm{A}$ and $\mathrm{B}$ compared to our original run (Fig. 7). Interestingly, the high altitude region of Joule heating in region $\mathrm{C}$ is again present, but it is now associated with thermalisation of kinetic energy stored in super-corotational rather than sub-corotational winds.

The equatorial temperature of $\sim 900 \mathrm{~K}$ (not shown) is now in approximate agreement with the observations (Seiff et al., 1998). The poleward convection of heat also slightly raises the temperature of the polar regions above $\sim 700 \mathrm{~K}$, which remains at the lower end of the range of polar $\mathrm{H}_{3}^{+}$temperature measurements (Lam et al., 1997; Stallard et al., 2002). Overall, therefore, this situation matches the temperature data well, producing temperatures reasonably in line with observations at all latitudes.

\subsubsection{Magnetospheric effects}

Figure 15 shows the parameters for the magnetosphere model in our standard format. These results are considerably different from those produced by previous runs. The thermosphere super-corotates everywhere within $30 R_{J}$, peaking at $\sim 20 \%$ super-corotation at a radius of about $15 R_{J}$. This large degree of super-corotation is enough for the magnetosphere to also super-corotate within $25 R_{J}$.

This has several peculiar effects on the values of $K$ and $\Sigma_{P}^{*}$. As the plasma velocity reaches perfect corotation, $K$ approaches negative infinity. $\Sigma_{P}^{*}$, which is a function of $(1-K)$, therefore approaches positive infinity. This radius is marked on the respective plots with a vertical dot-dash 

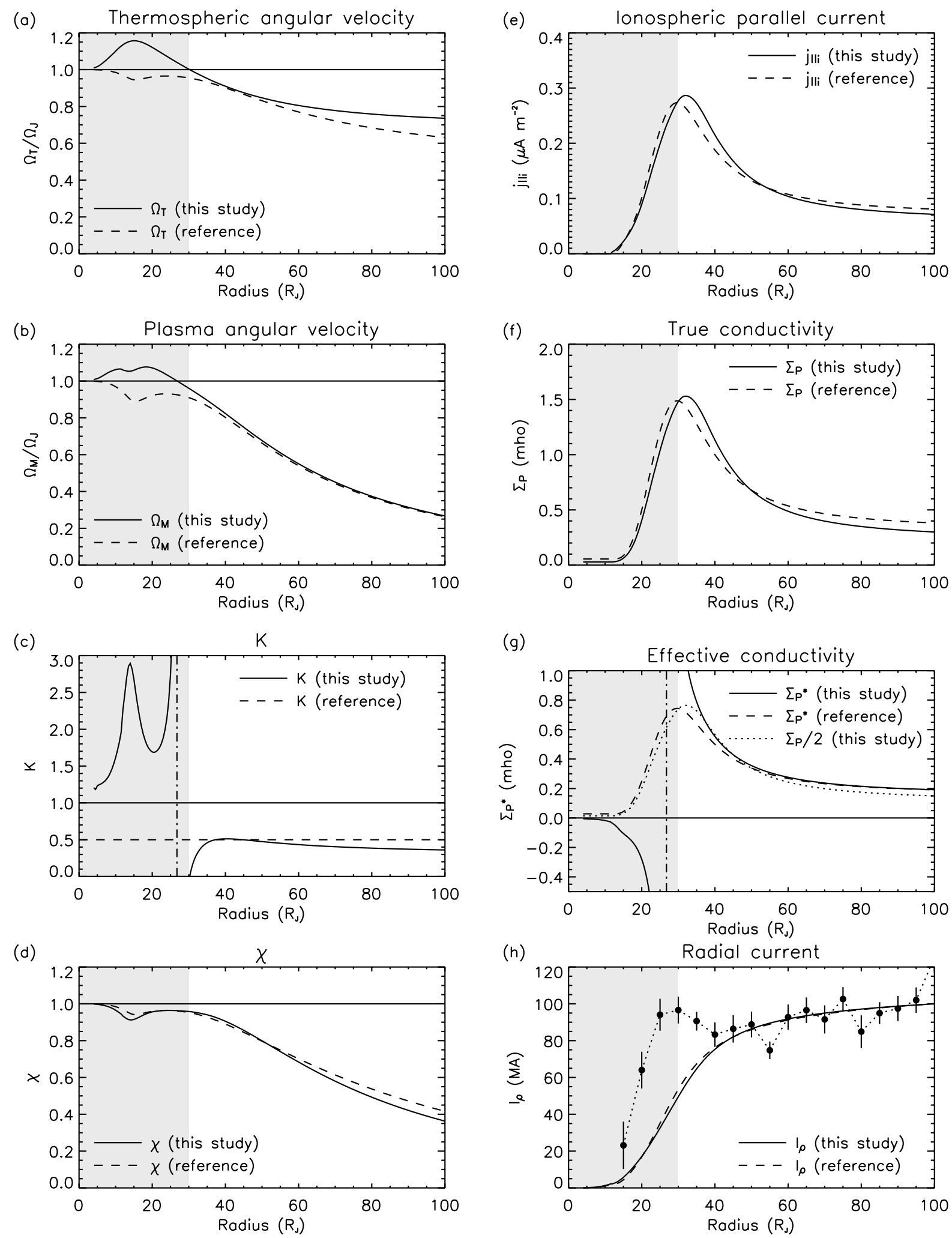

$$
E_{\text {fuc }}=1.0 \mathrm{~V} / \mathrm{m} \text { equatorial }
$$

Fig. 15. Parameters for r.m.s. fluctuations of $1.0 \mathrm{~V} / \mathrm{m}$ equatorward of $20^{\circ}$ colatitude, in the same format as Fig. 9 . Note that plots (c) and (g) now show negative values of $K$ and $\Sigma_{P}^{*}$ respectively. The radius at which these quantities tend to infinity and change sign is indicated by the vertical dash-dot line on each plot. This radius corresponds to the plasma being in perfect corotation with the planet. 
line. Within $25 R_{J}$, where the plasma super-corotates, $K$ is strongly positive and the effective conductivity is negative. Negative effective conductivity means that the atmospheric torque is tending to push the plasma velocity away from corotation - in this case towards greater super-corotation.

This peculiar behaviour is just a consequence of the definitions of these quantities, which were designed for a situation in which the plasma and neutrals exclusively sub-corotated. The behaviour of the conventional physical quantities is perfectly normal - the currents and true conductivities are very similar to those generated by our initial run (Fig. 9). These results demonstrate clearly the limited usefulness of the effective conductivity as a meaningful physical parameter in circumstances where super-corotation of the thermosphere is probable.

Introducing the equatorial energy source has thus had very little effect on the magnetosphere-atmosphere coupling currents. However, the $\sim 10 \%$ super-corotation of the plasma that this model predicts is in contradiction to the evidence (McNutt et al., 1979) which clearly shows sub-corotation of the plasma in the magnetosphere. To bring the plasma rotation velocity back in line with the reference model - and thus in line with the observations - would require a reduction in the true ionospheric conductivity in the inner region. As we have already discussed, such a modification would probably have a negligible effect on the thermospheric rotation velocity in the inner region, since this is controlled not by ion drag but by Coriolis acting on the meridional pressure gradient-driven poleward winds. Our results thus imply that if an equatorial heat source is responsible for the high thermospheric temperatures, the true conductivities around the equatorward edge of the main auroral oval must be smaller than previously anticipated.

Furthermore, looking at the plasma angular velocity curve in Fig. 15b, it is clear that the plasma angular velocity begins to diverge from the reference model at around $40 R_{J}$, and when the value of the background conductivity becomes important at around $15 R_{J}$ the plasma is already supercorotating by $\sim 5 \%$, in contradiction to observations. In this range of radii it is the aurorally enhanced conductivity that dominates, not the background. To match the data in this region we would thus have to reduce significantly the magnitude of the conductivity enhancement in the auroral oval.

\section{Discussion}

\subsection{Magnetosphere}

It seems clear that there is an important distinction to be drawn between the behaviour of the magnetosphere within and beyond $30 R_{J}$. The outer region is apparently relatively unaffected by the behaviour of the thermosphere, exhibiting behaviour very close to that of the reference model in all of the experiments described. In contrast, the inner region is very sensitive to the details of the thermospheric structure. Our results imply that the rotation of the inner magnetosphere must depend on two factors that have not previously been considered significant: the detailed dynamics of the thermosphere and the plasma flows in the middle and outer magnetosphere.

The dependence on thermospheric dynamics is clear from our results. We have shown model results for three different distributions of thermospheric heating, with no change in the nature of the rotational forcing from the magnetosphere. For each of these sets of results it is clear that the changes in the thermospheric dynamics driven by changes in the thermospheric heating distribution control the rotation rate of the inner magnetosphere. This is a different perspective to that implied by the model of Huang and Hill (1989), in which the thermosphere responds passively to driving from the marginal sub-corotation of the inner magnetosphere. Our results show that internal thermospheric dynamics are more important in this region: the degree of corotation of the magnetosphere is then determined from the value of the background conductivity.

The dependence on the middle and outer magnetospheres is less clear. However, as commented in Sect. 7.3, the supercorotation of the thermosphere connected to the inner magnetosphere is ultimately driven by the sub-corotation of the middle and outer magnetospheres and the thermospheric dynamics that this drives. Thus an increase or decrease in the sub-corotation of the middle and outer magnetospheres is expected, via the thermosphere, to cause an increase or decrease in the rotation velocity of the inner magnetosphere. Thus the thermosphere is expected to mediate an interaction between regions of the magnetosphere which are otherwise unconnected.

This predicted anti-correlation between the rotation velocities of the outer and inner magnetospheres deserves an explanation. This can be achieved by revisiting the framework used by Smith et al. (2007) to interpret the results of their Saturn model. By considering the hydrostatic equilibrium of the polar regions, they showed that this was perturbed by strong sub-corotational winds such that the thermosphere "collapsed" inwards towards the pole, drawing in gas from lower latitudes which were thus convectively cooled. The super-corotational winds equatorward of the region subject to significant ion drag were then generated by Coriolis forces acting on this poleward flowing gas.

It is clear from this interpretation that increasing the subcorotation of the middle and outer magnetospheres will in turn increase the sub-corotation of the polar thermosphere; the speed of the poleward winds; the magnitude of the Coriolis force acting on those winds; and thus the magnitude of the thermospheric super-corotation at latitudes connected to the inner magnetosphere. Due to the relatively low moment of inertia of the inner magnetosphere it is then brought almost into corotation with these super-corotating winds. 
From a global perspective, an increase in sub-corotation in the outer regions of the magnetosphere leads to a greater quantity of angular momentum being drawn from the planet. This increase in angular momentum flux through the system as a whole leads to a build-up of angular momentum in regions where the flux happens to be convergent but where there are few efficient sinks of angular momentum. One of these regions is the inner magnetosphere and connected thermosphere.

\subsection{Thermosphere}

It is worth emphasising that our results for the thermal structure at all latitudes remain very uncertain since there is only one reliable temperature profile (Seiff et al., 1998). This is insufficient to fully validate the magnitude of the meridional temperature and pressure gradients that are important in driving the global circulation. However, our results can still provide some useful pointers as to the origin of the high $(\sim 900 \mathrm{~K})$ low latitude temperatures.

Principally, our experiments seem to rule out the possibility that high latitude heating can explain the low latitude temperatures. This is consistent with recent results for Saturn (Smith et al., 2005b; Müller-Wodarg et al., 2006; Smith et al., 2007). Even when we added enough high latitude heating to raise the polar temperature to $1500 \mathrm{~K}$ - slightly in excess of the observations - the equatorial temperature was still low, in the region of $400 \mathrm{~K}$. Figure 16 shows a comparison between temperature profiles predicted by our model and the Galileo probe temperature profile (Seiff et al., 1998). The solid line shows the Galileo profile, the dashed line our predicted temperature at the equator when we apply low-latitude fluctuation heating, and the dotted line the predicted temperature at $45^{\circ}$ when we apply high latitude fluctuation heating.

There are two important distinctions between the dashed line (showing a temperature profile generated by local heating) and the dotted line (showing a temperature profile generated by redistributed heating). Firstly, as already mentioned, the redistributed heating only generates a profile comparable to the Galileo probe profile at $45^{\circ}$ latitude. This observation alone is almost sufficient to rule out redistribution from the poles as an energy source for low latitudes.

However, considering a situation in which redistribution from the poles did generate a temperature profile at the equator such as that shown by the dotted line, we can make a second observation. This is that the curvature of the profile generated by local heating is greater than that of the profile generated by redistributed heating, and in this sense is much closer to the data.

The curvature of the temperature profile (the second derivative of temperature $T$ with respect to altitude $z$ ) is related to the divergence of the vertical conductive heat flux $F_{\kappa}$ :

$\frac{\partial F_{\kappa}}{\partial z}=-\frac{\partial}{\partial z}\left(\kappa \frac{\partial T}{\partial z}\right)$

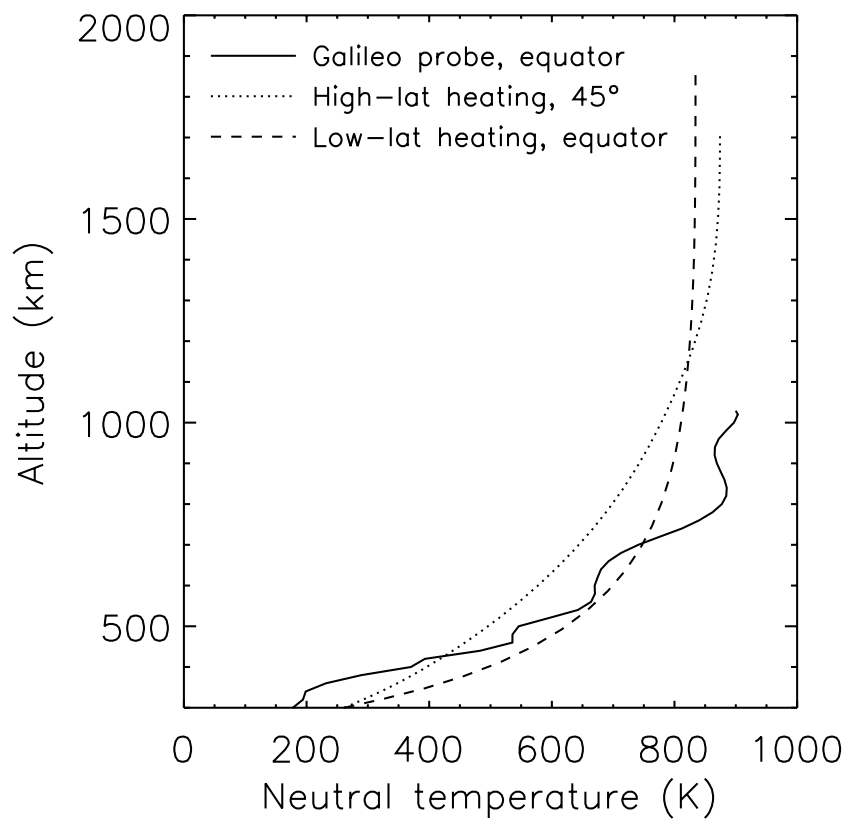

Fig. 16. Comparison between Galileo probe equatorial temperature profile (solid line; Seiff et al., 1998) and our results. The dashed line shows the equatorial temperature profile predicted when lowlatitude fluctuation heating is applied to our model. The dotted line shows the temperature profile at $45^{\circ}$ latitude predicted when highlatitude fluctuation heating is applied.

where $\kappa$ is the combined molecular and eddy thermal conductivity. A positive divergence of the heat flux must be balanced by an input of thermal energy, so a greater curvature corresponds to a greater input of thermal energy at that altitude. Both the dotted and dashed profile exhibit roughly the same temperature change between the mesopause and exosphere, but the tighter curvature of the dashed profile at low altitudes means that the energy input is concentrated within a more limited range of altitudes.

Since we use the same conductivity profile at all latitudes in our model, the original thermal energy input due to fluctuation heating has the same distribution with respect to pressure at all latitudes. A difference in the relative altitude of the original energy input can thus not account for the different curvatures of the two profiles. Rather, it seems that the profile produced by redistribution of heat energy has been "washed out" during transport by the action of vertical thermal conduction. This "washing out" of the curvature seems inevitable during meridional transport, since thermal conduction is a diffusive process that acts continuously to remove vertical temperature gradients. Since the equatorial data exhibits significant curvature, this indicates a concentrated energy input - and so it seems unlikely that it could be explained by redistribution.

We commented in Sect. 8 that results from the lowlatitude heating run should not be over-interpreted close to 
the equator due to the uncertainty in the accuracy of our conductivity model at these latitudes. We wish therefore to emphasise the general conclusion that local heating produces a tighter curvature in the temperature profile that, in terms of its general shape, is more consistent with observations. By contrast, the specific observation that the equatorial thermal profile produced by local heating is very similar to the Galileo probe profile shown in Fig. 16 should not be considered a significant result.

While the low-latitude fluctuation heating seems effective in approximately reproducing the observed equatorial temperatures, it has the clear disadvantage that it produces a super-corotating inner magnetosphere. This is caused by the super-corotating poleward winds generated by the poleward pressure gradient. If low-latitude heating is the solution, we must either have a much reduced ionospheric conductivity such that the magnetosphere does not super-corotate, or there must also be some extra heating in the polar regions to balance out the poleward pressure gradient. Our results thus tentatively imply that the high temperatures are due to neither a low nor a high latitude energy source alone, but are generated either by an energy source with a roughly uniform global distribution or by some mixture of sources acting at different latitudes.

Finally, we briefly discuss the effects on the thermospheric circulation of a less stagnant polar cap flow such as might be implied by an alternative model of the outer magnetosphere. A less stagnant (more rapidly rotating) flow in regions A and B would reduce the strength of the ion drag to which these regions are subjected. The structure of this region is determined by ion drag, which, through the action of Coriolis forces on westward winds, is ultimately responsible for driving the poleward circulation. Lower ion drag would almost certainly reduce the strength of this poleward circulation. This would lesson the intensity of both the hotspot at the pole and the cool region equatorward of the main oval described in Sect. 7. Since this latter feature is also linked to the super-corotating winds connected to the inner magnetosphere, the intensity of these winds would presumably also be reduced. We postpone a detailed study of these effects to a future paper.

\subsection{Ionospheric conductivity}

One omission that has already been discussed is that our conductivity profile is identical at all latitudes. This is important because different processes operating at different latitudes generate ionisation with different vertical distributions. For example, solar produced ionisation may be broadly distributed across a wide altitude range, while ionisation produced by hard particle precipitation may be formed in a relatively thin layer at a relatively low altitude.

As we have established, the rotation velocity $\Omega_{T}$ is both a combination of meridional and zonal components and a weighted average with altitude. The weighted average is de- termined by the conductivity profile. In effect, the altitude of the peak conductivity determines the altitude that the magnetosphere interacts with.

It is interesting to speculate on the consequences of this. It is clear that due to the importance of meridional winds the profile of neutral rotation velocity from our model varies smoothly with latitude. However, we always sample the same pressure level because our conductivity profile has the same vertical distribution at all latitudes. If the peak conductivity was at a deeper pressure level in the main auroral oval due to the intense particle precipitation in that narrow band of latitudes, then we would expect the rotation velocity at this deeper level to dominate $\Omega_{T}$. We might then witness a sharp change in $\Omega_{T}$ at the boundaries of the auroral oval.

Hence small-scale structure in the conductivity distribution could make it appear that there was small-scale structure in the neutral winds, even if in practice the winds were smoothly varying. Such structure could exist in principle on the smallest of scales.

\section{Conclusions}

To our knowledge the model described in this paper is the first to describe thermospheric and magnetospheric rotation velocities using coupled models that incorporate both radial (in the magnetosphere) and meridional (in the thermosphere) transport of angular momentum. Previous modelling studies for the thermosphere (Achilleos et al., 1998; Bougher et al., 2005) have not used self-consistent models of the magnetosphere, and previous magnetosphere models have at best incorporated localised models of vertical angular momentum transport in the thermosphere (Huang and Hill, 1989; Pontius, 1995).

The most important simplifications that we have made in order to develop a tractable model are as follows:

1. We have assumed axisymmetry of the thermosphere and magnetosphere. As discussed in Sect. 4.2 we believe that this introduces errors of less than about $20 \%$ to our calculations of thermospheric dynamics.

2. We have neglected the effect of parallel electric fields in decoupling the plasma flows in the equatorial magnetosphere and ionosphere.

3. We employ a highly simplified fixed conductivity model which implies an identical vertical distribution of conductivity at each latitude. This was necessary in order to simplify the integration of our magnetosphere model. Since the conductivity model is based on an auroral ionosphere model we believe it to be reasonably reliable at high latitudes where electron precipitation is likely to be the dominant ionisation process. At mid latitudes it is much less reliable, but, as discussed in Sect. 5.3, this is compensated for by the relatively low importance of Joule heating and ion drag at mid latitudes. 
Thus we have been able to examine for the first time the rotational coupling between the two systems on a non-local scale. We can divide our conclusion into two groups.

First, the general physical processes already reported by Smith et al. (2007) and Smith and Aylward (2008) in the context of Saturn, which we expected to apply to Jupiter:

1. Meridional advection is a much more important mechanism than viscosity for the supply of angular momentum to the thermosphere.

2. Super-corotation of the neutral atmosphere may arise at latitudes just equatorward of those coupled to subcorotating plasma in the magnetosphere. The supercorotation is ultimately driven by the sub-corotation of the middle and outer magnetospheres.

3. Mean field Joule heating and ion drag in the polar regions do not provide the energy that heats the equatorial regions to $\sim 900 \mathrm{~K}$. Instead, a hotspot is generated at the pole by meridionally convergent winds, and low latitudes are marginally cooled.

The generality of these processes to both gas giant planets has been demonstrated by this study, with the caveat that inputs omitted by our study may alter the magnitude of these effects. Our second group of conclusions consists of specific insights into the coupling between the thermosphere and magnetosphere that have been made possible by the unique nature of our model.

1. The rotation velocity of the thermosphere at latitudes coupled to the magnetosphere within $30 R_{J}$ is not directly controlled by the magnetosphere. Conversely, the rotation of this region of the magnetosphere is very sensitive to the structure of the connected thermosphere and ionosphere and, as discussed in Sect. 9.1, is indirectly influenced by the rotation of more distant regions of the magnetosphere.

2. A large additional heat source in the polar regions tends to reduce the rotation velocity of both the thermosphere and middle magnetosphere, through the action of Coriolis forces on equatorward flowing gas. The degree of equatorward redistribution is insufficient to explain the equatorial temperatures, and the low latitude temperature profiles that are generated do not show a good match with the curvature of the Galileo probe temperature profile.

3. An additional heat source, broadly distributed across the equatorial regions, can generate a good match with the observed equatorial temperatures. It also generates poleward flowing winds that produce substantial supercorotation at latitudes coupled to the inner magnetosphere, which in turn results in super-corotation of the inner magnetosphere, in contradiction to the observations. In this case our model either overestimates the auroral conductivity enhancements, or underestimates the heating in the polar region.

These conclusions all demonstrate a subtle and sensitive coupling between the three components of the system - magnetosphere, ionosphere and thermosphere. Our approach allows us to examine one question - possible solutions to the thermospheric energy crisis - and interpret the plausibility of these solutions in terms of ionospheric and magnetospheric parameters. For example, we can only accept an equatorial energy source if we are able to also match the associated plasma angular velocities to the data.

The model thus provides extra constraints on the behaviour of the thermosphere, for which very little data is available. If models of the thermosphere must also demonstrate reasonable consistency with the behaviour of the magnetosphere then we have an extra constraint on their plausibility.

Finally, the model demonstrates clearly that naively neglecting the thermosphere does not serve magnetosphere research well. The assumption that the thermosphere simply responds linearly to the magnetospheric sub-corotation is clearly inadequate, and the variety of behaviours exhibited by the thermospheric winds depending on the nature and distribution of the thermospheric heating shows that the thermosphere is a rich and complex aspect of the system.

\section{Appendix A}

\section{Details of middle magnetosphere model}

In this section we summarise the theoretical basis of Eqs. (15) and (16), which constitute our middle magnetosphere model. For full details the reader is referred to Nichols and Cowley (2004).

\section{A1 Magnetic field model}

As mentioned in Sect. 3.2, a flux function $F$ is used to define each axially symmetric shell of field line. In the ionosphere, where $B_{i}=2 B_{J}$, this is just given by $F_{i}=B_{J} \rho_{i}^{2}$. In the equatorial magnetosphere the vertical magnetic field $B_{z e}$ and flux function $F_{e}$ are given by the following expressions:

$$
\begin{aligned}
& B_{z e}\left(\rho_{e}\right)=-\left\{B_{o}\left(\frac{R_{J}}{\rho_{e}}\right)^{3} \exp \left[-\left(\frac{\rho_{e}}{\rho_{e o}}\right)^{5 / 2}\right]+A\left(\frac{R_{J}}{\rho_{e}}\right)^{m}\right\} \\
& F_{e}\left(\rho_{e}\right)=F_{\infty}+\frac{B_{o} R_{J}^{3}}{2.5 \rho_{e o}} \Gamma\left[-\frac{2}{5},\left(\frac{\rho_{e}}{\rho_{e o}}\right)^{5 / 2}\right]+\frac{A}{m-2}\left(\frac{R_{J}}{\rho_{e}}\right)^{m-2}
\end{aligned}
$$

where $\quad B_{o}=3.335 \times 10^{5} \mathrm{nT}, \quad \rho_{e o}=14.501 R_{J}$, $A=5.4 \times 10^{4} \mathrm{nT}, \quad m=2.71, \quad F_{\infty} \simeq 2.841 \times 10^{4} \mathrm{nT} R_{J}^{2} \quad$ and $\Gamma(a, z)=\int_{z}^{\infty} t^{a-1} e^{-t} d t$ is the incomplete gamma function. Note that the second expression is derived from the first by integration. For further discussion of the origin of these expressions the reader is referred to Nichols and 
Cowley (2004). As already mentioned in Sect. 6, we use $R_{J}^{N C}=71323 \mathrm{~km}$ in these formulae, consistent with Nichols and Cowley (2004). In all subsequent calculations, we employ Jupiter's equatorial radius, $R_{J}=71492 \mathrm{~km}$.

\section{A2 Field-aligned current}

As already described in Sect. 2, the difference between the plasma rotation velocity $\Omega_{M}$ and the effective thermospheric rotation velocity $\Omega_{T}$ drives an equatorward current $J_{\theta}$ in the ionosphere:

$J_{\theta}=\Sigma_{P} \rho_{i}\left(\Omega_{T}-\Omega_{M}\right) B_{i}=2 \Sigma_{P} \rho_{i}\left(\Omega_{T}-\Omega_{M}\right) B_{J}$

where $\Sigma_{P}$ is the true height-integrated Pedersen conductivity and $\rho_{i}$ is the perpendicular off-axis distance. Note that this is our notation for Eq. (5) of Nichols and Cowley (2004).

Comparing to the radial current $J_{\rho}$ in the magnetically connected equatorial magnetosphere, current continuity implies that:

$\rho_{e} J_{\rho}=2 \rho_{i} J_{\theta}$

where $\rho_{e}$ is the radial distance in the magnetosphere that maps to $\rho_{i}$ in the ionosphere. This is our notation for Eq. (8) of Nichols and Cowley (2004). The factor of 2 on the right hand side arises because the radial current in the magnetosphere closes the circuit for both hemispheres. Substituting Eq. (A2) into Eq. (A3), integrating in azimuth, and noting that on each flux shell $B_{J} \rho_{i}^{2}=F_{i}=F_{e}$ we obtain the following expression for the total azimuth-integrated radial current $I_{\rho}$ :

$I_{\rho}=8 \pi \Sigma_{P} F_{e}\left(\Omega_{T}-\Omega_{M}\right)$

which is our notation for Eq. (10) of Nichols and Cowley (2004).

The field-aligned current in the ionosphere $j_{\| i}$ is then calculated from the divergence of $I_{\rho}$ providing us with Eq. (16), which we repeat here:

$j_{\| i}=\frac{4 B_{J}}{\rho_{e}\left|B_{z e}\right|} \frac{d}{d \rho_{e}}\left[\Sigma_{P} F_{e}\left(\Omega_{T}-\Omega_{M}\right)\right]$

which is our notation for Eq. (12) of Nichols and Cowley (2004).

\section{A3 Angular momentum}

The second component of the magnetosphere model is the application of Newton's second law to the steady outward flow of plasma from the Io torus:

$$
\frac{d}{d \rho_{e}}\left(\dot{M} \rho_{e}^{2} \Omega_{M}\right)=\rho_{e} J_{\rho}\left|B_{z e}\right|
$$

which is our notation for Eq. (13) of Nichols and Cowley (2004). Here the LHS is the divergence of the outward plasma angular momentum flux, which is balanced by the RHS representing the azimuth-integrated input of angular momentum from the upper atmosphere. The quantity $\dot{M}=1000 \mathrm{~kg} \mathrm{~s}^{-1}$ is the plasma mass-loading rate in the Io torus, which is assumed to be constant.

Substituting Eq. (A4) into Eq. (A6) we obtain Eq. (15), which we repeat here:

$\frac{1}{\rho_{e}} \frac{d}{d \rho_{e}}\left(\rho_{e}^{2} \Omega_{M}\right)=\frac{8 \pi \Sigma_{P} F_{e}\left|B_{z e}\right|}{\dot{M}}\left(\Omega_{T}-\Omega_{M}\right)$

which is our notation for Eq. (14) of Nichols and Cowley (2004).

\section{Appendix B}

\section{Ionospheric conductivities}

The following expressions are used to calculate the Pedersen and Hall conductivities $\sigma_{P i}$ and $\sigma_{H i}$ due to a particular ion $i$ :

$\sigma_{P i}=\frac{e n_{i}}{|B|}\left[\frac{1}{r_{i}+r_{i}^{-1}}\right]$

$\sigma_{H i}=\frac{e n_{i}}{|B|}\left[\frac{r_{i}}{r_{i}+r_{i}^{-1}}\right]$

where $e$ is the electronic charge, $B$ is the magnetic flux density, $n_{i}$ is the ion number density, and $r_{i}=v_{i n} / \Omega_{i}$ is the ratio of the ion-neutral collision frequency $v_{i n}$ to the ion gyrofrequency $\Omega_{i}$. We calculate $v_{i n}$ using the expression given by Banks and Kockarts (1973):

$v_{i n}=2.6 \times 10^{-15} n_{n} \sqrt{\frac{\alpha_{0}}{\mu_{\text {in }}}}$

where $\alpha_{0}$ is the polarisability of the neutral gas (in units of $10^{-30} \mathrm{~m}^{3}$ ), given as $0.82,0.667$ and 0.21 for $\mathrm{H}_{2}, \mathrm{H}$ and He respectively, $n_{n}$ is the number density of the neutral species in $\mathrm{m}^{-3}$, and $\mu_{i n}$ is the reduced mass of the neutral and ionised species in atomic mass units. For simplicity we assume that the magnetic field is radial and constant.

Acknowledgements. The simulations in this study were performed using the HiPerSPACE facility at UCL, funded by the UK Particle Physics and Astronomy Research Council (PPARC). CGAS acknowledges receipt of a CASE studentship funded by PPARC and Sun Microsystems Ltd.

Topical Editor I. A. Daglis thanks two anonymous referees for their help in evaluating this paper.

\section{References}

Achilleos, N., Miller, S., Tennyson, J., Aylward, A. D., MuellerWodarg, I., and Rees, D.: JIM: A time-dependent, threedimensional model of Jupiter's thermosphere and ionosphere, J. Geophys Res., 103, 20089-20112, 1998. 
Atreya, S. K.: Atmospheres and Ionospheres of the Outer Planets and their Satellites, Springer-Verlag, 1986.

Banks, P. M. and Kockarts, G.: Aeronomy, Academic Press, 1973.

Bougher, S. W., Waite, J. H., Majeed, T., and Gladstone, G. R.: Jupiter Thermospheric General Circulation Model (JTGCM): Global structure and dynamics driven by auroral and Joule heating, J. Geophys. Res., 110, 4008, doi:10.1029/2003JE002230, 2005.

Codrescu, M. V., Fuller-Rowell, T. J., and Foster, J. C.: On the importance of E-field variability for Joule heating in the highlatitude thermosphere, Geophys. Res. Lett., 22, 2393-2396, 1995.

Connerney, J. E. P., Acuña, M. H., Ness, N. F., and Satoh, T.: New models of Jupiter's magnetic field constrained by the Io flux tube footprint, J. Geophys Res., 103, 11929-11940, 1998.

Cowley, S. W. H. and Bunce, E. J.: Origin of the main auroral oval in Jupiter's coupled magnetosphere-ionosphere system, Planetary \& Space Science, 49, 1067-1088, 2001.

Cowley, S. W. H. and Bunce, E. J.: Corotation-driven magnetosphere-ionosphere coupling currents in Saturn's magnetosphere and their relation to the auroras, Ann. Geophys., 21, 1691-1707, 2003, http://www.ann-geophys.net/21/1691/2003/.

Cowley, S. W. H., Bunce, E. J., Stallard, T. S., and Miller, S.: Jupiter's polar ionospheric flows: Theoretical interpretation, Geophys. Res. Lett., 30, 24-1, doi:10.1029/2002GL016030, 2003

Cowley, S. W. H., Alexeev, I. I., Belenkaya, E. S., Bunce, E. J., Cottis, C. E., Kalegaev, V. V., Nichols, J. D., Prange, R., and Wilson, F. J.: A simple axisymmetric model of magnetosphereionosphere coupling currents in Jupiter's polar ionosphere, J. Geophys Res., 110, A11209, doi:10.1029/2005JA011237, 2005.

Cowley, S. W. H., Badman, S. V., Imber, S. M., and Milan, S. E.: Comment on "Jupiter: A fundamentally different magnetospheric interaction with the solar wind" by D. J. McComas and F. Bagenal, Geophys. Res. Lett., 35, 10101, doi:10.1029/2007GL032645, 2008.

Delamere, P. A. and Bagenal, F.: Modeling variability of plasma conditions in the Io torus, J. Geophys. Res. (Space Physics), 108, 1276, doi:10.1029/2002JA009706, 2003.

Gladstone, R., Majeed, T., Bougher, S., Waite, H., and Clarke, J.: Using HST-STIS Observations of Auroral Ly $\alpha$ Line Profiles to Map High-Altitude Winds on Jupiter, AGU Spring Meeting Abstracts, pp. A2+, 2005.

Grodent, D., Waite, J. H., and Gérard, J.: A self-consistent model of the Jovian auroral thermal structure, J. Geophys Res., 106, 12933-12952, 2001.

Grodent, D., Clarke, J. T., Kim, J., Waite, J. H., and Cowley, S. W. H.: Jupiter's main auroral oval observed with HST-STIS, J. Geophys Res., 108, 2-1, doi:10.1029/2003JA009921, 2003.

Hill, T. W.: Inertial limit on corotation, J. Geophys Res., 84, 6554 6558, 1979.

Hill, T. W.: Corotation lag in Jupiter's magnetosphere - Comparison of observation and theory, Science, 207, 301-302, 1980.

Hill, T. W.: The Jovian auroral oval, J. Geophys Res., 106, 8101$8108,2001$.

Hinson, D. P., Flasar, F. M., Kliore, A. J., Schinder, P. J., Twicken, J. D., and Herrera, R. G.: Jupiter's ionosphere: Results from the first Galileo radio occultation experiment, Geophys. Res. Lett.,
24, 2107-2110, 1997.

Hinson, D. P., Twicken, J. D., and Karayel, E. T.: Jupiter's ionosphere: New results from Voyager 2 radio occultation measurements, J. Geophys Res., 103, 9505-9520, 1998.

Huang, T. S. and Hill, T. W.: Corotation lag of the Jovian atmosphere, ionosphere, and magnetosphere, J. Geophys Res., 94, 3761-3765, 1989.

Ingersoll, A. P., Dowling, T. E., Gierasch, P. J., Orton, G. S., Read, P. L., Sánchez-Lavega, A., Showman, A. P., Simon-Miller, A. A., and Vasavada, A. R.: Dynamics of Jupiter's atmosphere, pp. 105-128, Jupiter. The Planet, Satellites and Magnetosphere, 2004.

Isbell, J., Dessler, A. J., and Waite, J. H.: Magnetospheric energization by interaction between planetary spin and the solar wind, $\mathrm{J}$. Geophys Res., 89, 10716-10722, 1984.

Khurana, K. K.: Influence of solar wind on Jupiter's magnetosphere deduced from currents in the equatorial plane, J. Geophys Res., 106, 25999-26016, 2001.

Khurana, K. K., Kivelson, M. G., Vasyliunas, V. M., Krupp, N., Woch, J., Lagg, A., Mauk, B. H., and Kurth, W. S.: The configuration of Jupiter's magnetosphere, pp. 593-616, Jupiter. The Planet, Satellites and Magnetosphere, 2004.

Kivelson, M. G. and Southwood, D. J.: Dynamical consequences of two modes of centrifugal instability in Jupiter's outer magnetosphere, J. Geophys. Res. (Space Physics), 110, 12209, doi:10.1029/2005JA011176, 2005.

Krupp, N., Vasyliunas, V. M., Woch, J., Lagg, A., Khurana, K. K., Kivelson, M. G., Mauk, B. H., Roelof, E. C., Williams, D. J., Krimigis, S. M., Kurth, W. S., Frank, L. A., and Paterson, W. R.: Dynamics of the Jovian magnetosphere, pp. 617-638, Jupiter. The Planet, Satellites and Magnetosphere, 2004.

Lam, H. A., Achilleos, N., Miller, S., Tennyson, J., Trafton, L. M., Geballe, T. R., and Ballester, G. E.: A Baseline Spectroscopic Study of the Infrared Auroras of Jupiter, Icarus, 127, 379-393, 1997.

Lystrup, M. B., Miller, S., Dello Russo, N., Vervack Jr., R. J., and Stallard, T.: First Vertical Ion Density Profile in Jupiter's Auroral Atmosphere: Direct Observations using the Keck II Telescope, Astrophysical Journal, 677, 790-797, 2008.

Mauk, B. H., Anderson, B. J., and Thorne, R. M.: MagnetosphereIonosphere Coupling at Earth, Jupiter, and Beyond, pp. 97, Atmospheres in the Solar System: Comparative Aeronomy, 2002.

McComas, D. J. and Bagenal, F.: Jupiter: A fundamentally different magnetospheric interaction with the solar wind, Geophys. Res. Lett., 34, 20106, doi:10.1029/2007GL031078, 2007.

McComas, D. J. and Bagenal, F.: Reply to comment by S. W. H. Cowley et al. on "Jupiter: A fundamentally different magnetospheric interaction with the solar wind", Geophys. Res. Lett., 35, 10103, doi:10.1029/2008GL034351, 2008.

McNutt, R. L., Belcher, J. W., Sullivan, J. D., Bagenal, F., and Bridge, H. S.: Departure from rigid co-rotation of plasma in Jupiter's dayside magnetosphere, Nature, 280, 803, 1979.

Melin, H., Miller, S., Stallard, T., Smith, C., and Grodent, D.: Estimated energy balance in the jovian upper atmosphere during an auroral heating event, Icarus, 181, 256-265, 2006.

Miller, S., Achilleos, N., Ballester, G. E., Geballe, T. R., Joseph, R. D., Prange, R., Rego, D., Stallard, T., Tennyson, J., Trafton, L. M., and Waite, J. H.: The role of $\mathrm{H}_{3}^{+}$in planetary atmospheres, Royal Society of London Philosophical Transactions Series A, 
358, 2485-2502, 2000.

Millward, G., Miller, S., Stallard, T., Aylward, A. D., and Achilleos, N.: On the Dynamics of the Jovian Ionosphere and Thermosphere III: The Modelling of Auroral Conductivity, Icarus, 160, 95-107, 2002.

Müller-Wodarg, I. C. F., Mendillo, M., Yelle, R. V., and Aylward, A. D.: A global circulation model of Saturn's thermosphere, Icarus, 180, 147-160, 2006.

Nichols, J. and Cowley, S.: Magnetosphere-ionosphere coupling currents in Jupiter's middle magnetosphere: effect of precipitation-induced enhancement of the ionospheric Pedersen conductivity, Ann. Geophys., 22, 1799-1827, 2004, http://www.ann-geophys.net/22/1799/2004/.

Pontius, D. H.: Implications of variable mass loading in the Io torus: The Jovian flywheel, J. Geophys Res., 100, 19531-19540, 1995.

Pontius, D. H.: Radial mass transport and rotational dynamics, J. Geophys Res., 102, 7137-7150, 1997.

Richardson, J. D.: Thermal ions at Saturn - Plasma parameters and implications, J. Geophys Res., 91, 1381-1389, 1986.

Richardson, J. D., Eviatar, A., McGrath, M. A., and Vasyliũnas, V. M.: OH in Saturn's magnetosphere: Observations and implications, J. Geophys Res., 103, 20245-20256, 1998.

Saur, J., Mauk, B. H., Kaßner, A., and Neubauer, F. M.: A model for the azimuthal plasma velocity in Saturn's magnetosphere, J. Geophys Res., 109, 5217, doi:10.1029/2003JA010207, 2004.

Seiff, A., Kirk, D. B., Knight, T. C. D., Young, R. E., Mihalov, J. D., Young, L. A., Milos, F. S., Schubert, G., Blanchard, R. C., and Atkinson, D.: Thermal structure of Jupiter's atmosphere near the edge of a $5-\mu \mathrm{m}$ hot spot in the north equatorial belt, J. Geophys Res., 103, 22857-22890, 1998.

Siscoe, G. L. and Summers, D.: Centrifugally driven diffusion of Iogenic plasma, J. Geophys Res., 86, 8471-8479, 1981.
Smith, C. G. A. and Aylward, A. D.: Coupled rotational dynamics of Saturn's thermosphere and magnetosphere: a thermospheric modelling study, Ann. Geophys., 26, 1007-1027, 2008, http://www.ann-geophys.net/26/1007/2008/.

Smith, C. G. A., Miller, S., and Aylward, A. D.: Magnetospheric energy inputs into the upper atmospheres of the giant planets, Ann. Geophys., 23, 1943-1947, 2005a, http://www.ann-geophys.net/23/1943/2005/.

Smith, C. G. A., Aylward, A. D., Miller, S., and Müller-Wodarg, I. C. F.: Polar heating in Saturn's thermosphere, Ann. Geophys., 23, 2465-2477, 2005b, http://www.ann-geophys.net/23/2465/2005/.

Smith, C. G. A., Aylward, A. D., Millward, G., Miller, S., and Moore, L. E.: An unexpected cooling effect in Saturn's upper atmosphere, Nature, 445, 399-401, 2007.

Stallard, T., Miller, S., Millward, G., and Joseph, R. D.: On the Dynamics of the Jovian Ionosphere and Thermosphere. II. The Measurement of $\mathrm{H}_{3}^{+}$Vibrational Temperature, Column Density, and Total Emission, Icarus, 156, 498-514, 2002.

Stallard, T. S., Miller, S., Cowley, S. W. H., and Bunce, E. J.: Jupiter's polar ionospheric flows: Measured intensity and velocity variations poleward of the main auroral oval, Geophys. Res. Lett., 30, 25-8, doi:10.1029/2002GL016031, 2003.

Su, Y.-J., Ergun, R. E., Bagenal, F., and Delamere, P. A.: Io-related Jovian auroral arcs: Modeling parallel electric fields, J. Geophys. Res. (Space Physics), 108, 1094, doi:10.1029/2002JA009247, 2003.

Waite, J. H., Cravens, T. E., Kozyra, J., Nagy, A. F., Atreya, S. K., and Chen, R. H.: Electron precipitation and related aeronomy of the Jovian thermosphere and ionosphere, J. Geophys Res., 88, 6143-6163, 1983. 